\title{
Fluoroscopic Characterization of Colonic Dysmotility Associated to Opioid and Cannabinoid Agonists in Conscious Rats
}

\begin{abstract}
Susana Díaz-Ruano, ${ }^{1}$ Ana E López-Pérez, ${ }^{1,5}$ Rocío Girón, ${ }^{2,3,4,5}$ Irene Pérez-García, ${ }^{2}$ María I Martín-Fontelles, ${ }^{2,3,4,5}$ and Raquel Abalo ${ }^{2,3,4,5 *}$
${ }^{1}$ Unidad de Dolor, Servicio de Anestesiología, Hospital General Universitario Gregorio Marañón, Madrid, Spain; ${ }^{2}$ Departamento de Ciencias Básicas de la Salud, Facultad de Ciencias de la Salud, Universidad Rey Juan Carlos, Alcorcón, Madrid, Spain; ${ }^{3}$ Unidad Asociada I+D+i al Instituto de Investigación en Ciencias de la Alimentación, CIAL (CSIC), Madrid, Spain; ${ }^{4}$ Unidad Asociada I+D+i al Instituto de Química Médica, IQM (CSIC), Madrid, Spain; and ${ }^{5}$ Grupo de Excelencia Investigadora URJC-Banco de Santander-Grupo Multidisciplinar de Investigación y Tratamiento del Dolor (i+DOL), Madrid, Spain
\end{abstract}

\section{Background/Aims}

Gastrointestinal adverse effects have a major impact on health and quality of life in analgesics users. Non-invasive methods to study gastrointestinal motility are of high interest. Fluoroscopy has been previously used to study gastrointestinal motility in small experimental animals, but they were generally anesthetized and anesthesia itself may alter motility. In this study, our aim is to determine, in conscious rats, the effect of increasing doses of 2 opioid (morphine and loperamide) and 1 cannabinoid (WIN 55,212-2) agonists on colonic motility using fluoroscopic recordings and spatio-temporal maps.

\section{Methods}

Male Wistar rats received barium sulfate intragastrically, 20-22 hours before fluoroscopy, so that stained fecal pellets could be seen at the time of recording. Animals received an intraperitoneal administration of morphine, loperamide, or WIN 55,212-2 (at 0.1, 1, 5 , or $10 \mathrm{mg} / \mathrm{kg}$ ) or their corresponding vehicles (saline, Cremophor, and Tocrisolve, respectively), 30 minutes before fluoroscopy. Rats were conscious and placed within movement-restrainers for the length of fluoroscopic recordings (120 seconds). Spatio-temporal maps were built, and different parameters were analyzed from the fluoroscopic recordings in a blinded fashion to evaluate colonic propulsion of endogenous fecal pellets.

\section{Results}

The analgesic drugs inhibited propulsion of endogenous fecal pellets in a dose-dependent manner.

\section{Conclusions}

Fluoroscopy allows studying colonic propulsion of endogenous fecal pellets in conscious rats. Our method may be applied to the noninvasive study of the effect of different drug treatments and pathologies.

(J Neurogastroenterol Motil 2019;25:300-315)

Key Words

Analgesics, opioids; Cannabinoids; Colonic motility; Fluoroscopy; Rats

Received: December 3, 2018 Revised: February 1, 2019 Accepted: February 12, 2019

(a) This is an Open Access article distributed under the terms of the Creative Commons Attribution Non-Commercial License (http://creativecommons. org/licenses/by-nc/4.0) which permits unrestricted non-commercial use, distribution, and reproduction in any medium, provided the original work is properly cited.

${ }^{*}$ Correspondence: Raquel Abalo, PhD

Departamento de Ciencias Básicas de la Salud, Facultad de Ciencias de la Salud, Universidad Rey Juan Carlos, Avda. de Atenas s/n, 28922 Alcorcón, Madrid, Spain

Tel: +34-91-488-8854, Fax: +34-91-488-8955, E-mail: raquel.abalo@urjc.es

Susana Díaz-Ruano and Ana E López-Pérez have contributed equally to this manuscript. 


\section{Introduction}

The digestive tract motor disorders associated with the administration of drugs commonly used to treat pain are difficult to manage. The use of opioids in the treatment of chronic pain has increased in the past decade. Gastrointestinal adverse effects have a major impact on health and quality of life in opioid users. ${ }^{1}$ Opioidinduced constipation is the most common gastrointestinal adverse effect, $^{2}$ with a rate from $5 \%$ to $97 \%,{ }^{3}$ causing a significant reduction in quality of life. ${ }^{4}$ Other analgesic drugs also alter gastrointestinal motor function, including cannabinoids, ${ }^{5}$ and even NSAID, independently from their inflammatory effect on gastrointestinal mucosa. ${ }^{6}$

Different methods have been used to evaluate gastrointestinal motility in experimental animals. The less invasive methods are more adequate to mimic the clinical situation, but anesthetics or sedative drugs are often used, particularly in small laboratory animals, and these drugs can interfere with gastrointestinal motility. ${ }^{7,8}$ Previously, radiographic methods have been used combined with a simple semiquantitative analysis that allow the use of conscious rats to study the effect of different drugs administered either acutely or chronically, including opioids ${ }^{9}$ and cannabinoids, ${ }^{10,11}$ on general gastrointestinal motor function. Interestingly, spatio-temporal maps (STM), similar to those used previously to characterize motor function of isolated segments of the gut in organ bath experiments, ${ }^{12-14}$ were applied to analyze small intestinal contractility from fluoroscopic recordings obtained in vivo from conscious rats. ${ }^{15}$ Furthermore, in a recent study of the effect of the antitumoral drug vincristine on gastrointestinal motility, STM were used to represent colonic propulsion of endogenous fecal pellets from fluoroscopic recordings. ${ }^{16}$ Here, we extend these studies and evaluate the effect of different analgesics on colonic propulsion of endogenous fecal pellets in non-anesthetized rats.

The analgesics selected were morphine, loperamide, and the cannabinoid WIN 55,212-2. Morphine is a major opioid agonist commonly used in the clinic for the relief of moderate to severe pain. ${ }^{17}$ Loperamide is a synthetic opioid used in the clinic to treat diarrhea. ${ }^{18}$ At the therapeutic oral doses used for this indication, loperamide is devoid of central effects. ${ }^{19}$ In fact, loperamide is not clinically used as an analgesic, but it was recently demonstrated to produce peripheral analgesia in experimental animals when intraperitoneally administered. ${ }^{20}$ WIN $55,212-2$ is a potent nonselective cannabinoid receptor agonist, with analgesic properties mediated through cannabinoid receptor type 1 (CB1) and CB2. ${ }^{21-23}$
These drugs are known to inhibit gastrointestinal motility. Whereas morphine and loperamide exert their constipating effects mainly through mu opioid receptors, ${ }^{24}$ WIN 55,212-2 does so mainly through CB1 cannabinoid receptors. ${ }^{5}$ Importantly, these receptors are present in the myenteric plexus, intrinsically responsible for gut motor function. $^{25}$

In this study, our aim is to determine the effect of increasing doses of morphine, loperamide, and WIN 55,212-2, on rat colonic endogenous fecal pellet propulsion using fluoroscopic recordings and STM. This method is non-invasive and can be applied without anesthesia.

\section{Materials and Methods}

The in vivo experiments were designed and performed in accordance with the European and Spanish legislation on care and use of experimental animals (EU Directive 2010/63/EU for animal experiments; R.D. 53/2013), were approved by the Ethic Committee at Universidad Rey Juan Carlos and Comunidad de Madrid (PROEX 061/18) and conducted in accordance with the Declaration of the National Institutes of Health Guide for Care and Use of Laboratory Animals. All efforts were made to minimize animal pain or discomfort.

\section{Animals and Protocol}

Sixty-four (morphine group: MOR), 51 (loperamide group: LOP), and 39 (WIN 55,212-2 group: WIN) male Wistar rats (200-400 g) were obtained from the Veterinary Unit of Hospital General Universitario Gregorio Marañón (HGUGM, Madrid, Spain), where the experiments were performed. Animals were group-housed (3-4/cage) in standard transparent cages $(60 \mathrm{~cm}$ $\times 40 \mathrm{~cm} \times 20 \mathrm{~cm}$ ), under environmentally controlled conditions (temperature, $20^{\circ} \mathrm{C}$; humidity, $60 \%$ ), with a 12-hour light/12-hour dark cycle. Animals had free access to standard laboratory rat chow (Harlan Laboratories Inc, Barcelona, Spain) and sterile tap water, were not fasted prior to loading with contrast medium or recording (see below) and were conscious during X-ray sessions. No anesthesia was used, so that gastrointestinal motility was not altered.

\section{Fluoroscopic Recordings of Gastrointestinal Motor Function}

For these experiments, 2 loads of barium sulfate $(1.5 \mathrm{~mL})$ (Barigraph AD; Juste SAQF, Madrid, Spain; $2 \mathrm{~g} / \mathrm{L}$, at $22^{\circ} \mathrm{C}$ ) were given intragastrically, through curved gavage needles (14 gauge, 2.9 inch length, and $4 \mathrm{~mm}$ ball diameter), in 2 different moments: 21.5 
hours (approximately) and 1.5 hours before fluoroscopy (Fig. 1). Thus, at the time of fluoroscopy, the first load of barium is generally seen as a relatively weak staining of the cecum and fecal pellets, and the second load of contrast strongly stains the stomach and part of the small intestine (but not the cecum). This procedure allows the recording of motor function of all gastrointestinal regions in just one fluoroscopy session per time-point and reduces exposure to radiation of both animals and researchers. In the present study, we focused on the fecal pellets stained by the first load of barium, whereas the second load of barium that stained the upper gastrointestinal tract (whose motor function is currently under analysis in our laboratory) allowed us to confirm that no anatomic or functional alteration was present in the rats studied that could have had an influence on our results for the colon (anatomy and transit in the stomach and small intestine were normal).

Fluoroscopy was performed as previously described with slight modifications. ${ }^{15,16}$ Plain facial radiographs and fluoroscopic videos of the gastrointestinal tract were obtained using a digital X-ray apparatus (Siremobil Compact L; Siemens, Erlangen, Germany; $60 \mathrm{kV}, 7 \mathrm{~mA}$ ) and captured with Elgato Video Capture software (Munich, Germany). Exposure time was 0.06 seconds for X-ray shots, and 120 seconds for fluoroscopic recordings. Immobilization of the rats in the prone position was achieved by placing them inside adjustable hand-made transparent plastic tubes. Rats did not receive anesthesia and were immobilized inside the plastic tubes. To reduce stress, rats were habituated to stay within the plastic device for the recording period and were released immediately after each X-ray shot or video recording (ie, immobilization lasted less than 2.5 minutes). An X-ray was taken immediately after the second load of barium to confirm staining of both proximal (stronger) and distal (weaker) parts of the gastrointestinal tract (barium staining the cecum at the time of the X-ray would eventually stain also the fecal pellets whose movement would be followed during fluoroscopy). Fluoroscopic videos were recorded at 25 frames/sec, 1.5 hours after the second load of barium, when several loops of the small intestine had filled with contrast medium but before the cecum had been reached with this second load of barium (this time-point was determined as adequate based on previous studies ${ }^{15}$ ). Rats received an intraperitoneal injection of the drug of interest or its vehicle 30 minutes before fluoroscopy. Drug doses and vehicles were as follows (Fig. 1): morphine at 0.1-10 mg/kg and saline (1 mL/kg); loperamide at $0.1-10 \mathrm{mg} / \mathrm{kg}$ and Cremophor (1 mL/kg); and WIN
A

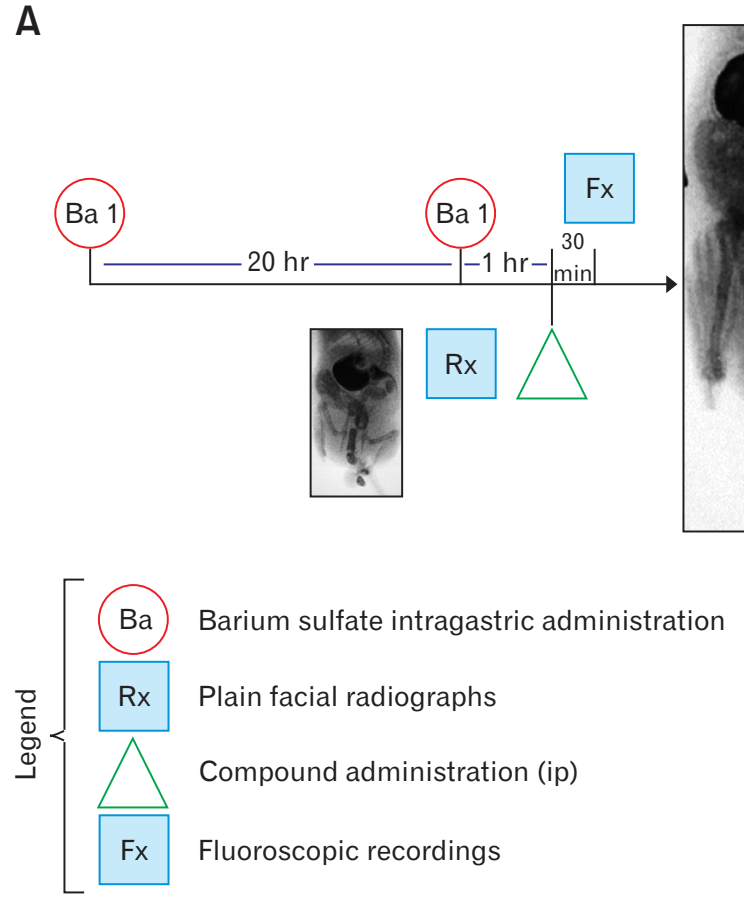

B

\begin{tabular}{|c|c|c|c|}
\hline & Saline & CREM & TOCRI \\
\hline 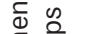 & MOR 0.1 & LOP 0.1 & WIN 0.1 \\
\hline$\overline{0}$ & MOR 1 & LOP 1 & WIN 1 \\
\hline 产 ప & MOR 5 & LOP 5 & WIN 5 \\
\hline & MOR 10 & LOP 10 & \\
\hline
\end{tabular}

Figure 1. Experimental protocol. (A) Two loads of barium sulfate (Ba) were administered per os in 2 different moments: 20-22 hours and 1.5 hours before fluoroscopy $(\mathrm{Fx})$. A plain radiograph $(\mathrm{Rx})$ was taken immediately after the second load of barium. Drugs were injected 1 hour after. Fluoroscopy was performed 30 minutes after the drugs or their vehicles. (B) Different experimental groups were used according to treatment and dose $(0.1,1,5$ or $10 \mathrm{mg} / \mathrm{kg}$ ) administered: morphine (MOR), loperamide (LOP), WIN 55,212-2 (WIN) or their corresponding vehicles (saline, Cremophor [CREM], and Tocrisolve [TOCRI], respectively). ip, intraperitoneal. 
$55,212-2$ at $0.1-5 \mathrm{mg} / \mathrm{kg}$ (for technical reasons, it was not possible to use WIN 55,212-2 at the dose of $10 \mathrm{mg} / \mathrm{kg}$ ) and Tocrisolve (1 $\mathrm{mL} / \mathrm{kg}$ ). The investigator was protected by lead shields for thyroid and abdomino-pelvic viscera, and remained behind a lead screen, at least 2 meters from the X-ray source, during image capture. At the end of the study, animals were sacrificed by overdose of anesthetic (pentobarbital).

\section{Analysis of the Recordings}

As already mentioned, here we analyzed the movement of the fecal pellets, stained by the first load of barium. Therefore, rats not showing any staining in the cecum or fecal pellets in the X-ray performed after the second barium load were not used in this particular study. Fluoroscopic videos in which movement of the rat did not allow for the analysis of at least 75 seconds were also discarded. Only videos displaying fecal pellets in the colon were further analyzed. Each fluoroscopic recording was broken down into 120 frames with QuickTime Pro (Apple Inc, Cupertino, CA, USA) and analyzed as follows (Fig. 2):

(1) The number of fecal pellets observed along the colon during the fluoroscopic video was recorded; their diameter was measured with Image J (free access software in www.imagej.net).

(2) For each bolus within the colon, the propulsion speed (PS) was measured as the distance travelled per time unit from its proximal position towards the anus, using the intervertebral disc placed at the root of the animal's tail (located at the level of the anal sphincter) as an anatomic reference (Fig. 2A).

(3) A STM was created with Excel to represent the progress of fecal pellets within the descending colon and rectum, using a binary code to represent the position of each fecal pellet along the colorectum: 0 = absent; $1=$ present (Fig. 2A). Staining of fecal pellets was generally too faint and their contrast too low against the background (due to the interference of the soft tissue and bones) to use available computerized tools to build diameter maps, but it was possible to follow their movement along the colon by eye, using a constant reference point for each pellet (distal end, proximal end, or middle point, depending on the particular pellet), and manually represent their position on the STM (see Supplementary material for further details about STM creation and examples of videos; Supplementary Fig. 1 shows 2 representative examples of STM, one from a rat with relatively well-stained fecal pellets, and another one from a rat whose fecal pellets were only faintly stained, as built from Supplementary Videos 1 and 2, respectively). From each STM, different parameters were obtained (Fig. 2B):

a. Total and mean number of fecal pellets (MFP) b. Percentage of pellets that exited the colon (FP exits)

c. Propulsion speed (PS') of each pellet, measured as the distance travelled (D') per time unit ( $\mathrm{T}^{\prime}$ ) from its initial position towards the anus, calculated from the STM.

d. For each pellet, maximum period of the recording without movement (maximum immobility time [MIT]).

(4) Fecal pellets as shown in the STM were categorized into 2 groups. Group 1 (slow pellets): pellets that moved 0-2.5 cm, and Group 2 (fast pellets): pellets that moved $\geq 2.5 \mathrm{~cm}$. Fecal pellets initially positioned $1 \mathrm{~cm}$ or $2 \mathrm{~cm}$ proximal to the anus were included in the fast category if they exited the colon in less than 60 seconds (half the duration of the movies); otherwise, they were included in the slow category.

(5) STM were categorized into 2 groups, according to the movement of their pellets (see point 4). Group 1 (slow STM): STM in which all fecal pellets were slow, and Group 2 (fast STM): STM in which at least one fecal pellet was fast.

(6) STM were categorized in 2 additional groups. Group 1: STM in which no fecal pellet exited the colon, and Group 2: STM showing at least one fecal pellet exiting the colon.

\section{Compounds and Drugs}

Barium sulfate (Barigraf AD; Juste SAQF) was suspended in tap water and continuously hand-stirred until administration. Morphine and loperamide were obtained from Laboratorios Abelló (Madrid, Spain) and dissolved in saline $(0.9 \% \mathrm{NaCl}$ in distilled water). Cremophor EL was obtained from Sigma-Aldrich (Madrid, Spain) and dissolved at 20\% in saline. WIN 55,212-2 (Tocris, Cookson, Bristol, UK) was dissolved in Tocrisolve, a commercially available water-soluble emulsion composed of a 1:4 ratio of soya oil/ water that is emulsified with the block co-polymer Pluronic F68 (Tocris; $30 \mu \mathrm{L}$ in $0.5 \mathrm{~mL}$ of saline solution).

\section{Statistical Methods}

Data are presented as the mean values \pm SEM. Differences between groups were analyzed using one-way ANOVA followed by post-hoc Bonferroni multiple comparison test, and $\chi^{2}$ test. Values of $P<0.05$ were regarded as being significantly different.

\section{Results}

Body weight recorded before the first load of barium was not significantly different ( $P>0.05$, one-way ANOVA) among the different experimental groups $(279 \pm 11 \mathrm{~g}$ with a range between 200-400 g). 
A

Frame 1

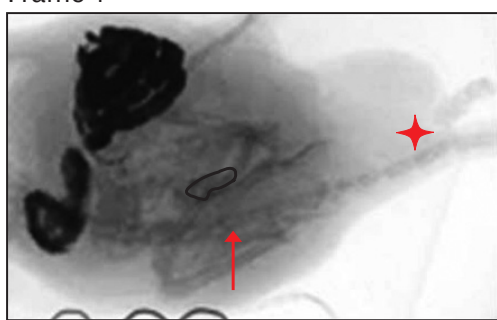

Oral $\longleftrightarrow$ Aboral
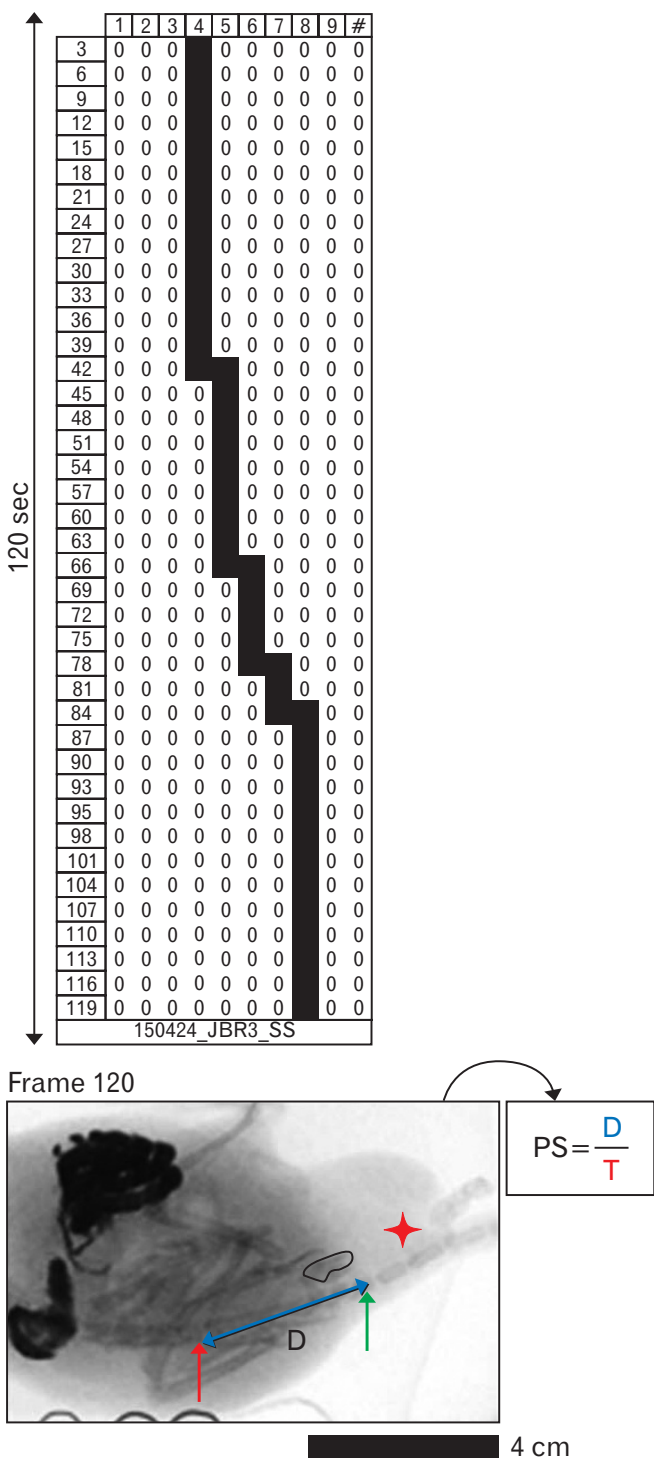

B

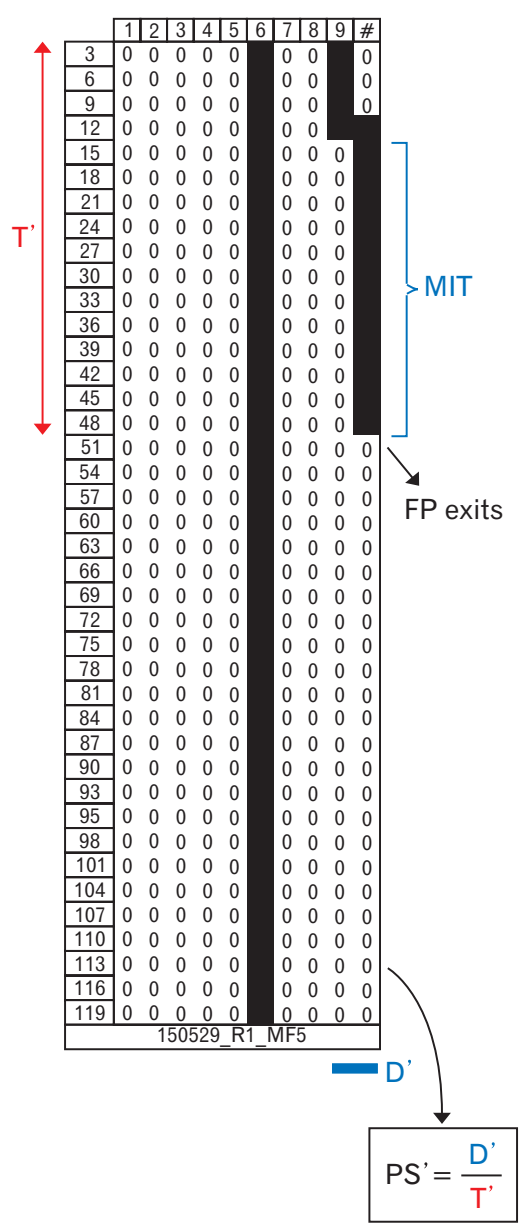

Figure 2. Analysis of the recordings. (A) Fluoroscopy recordings were broken down in 120 frames ( 1 frame/sec). In each frame, the intervertebral disc at the anus was identified and used as reference (star). Fecal pellet movement was represented in spatio-temporal maps (STM) built in Excel. In each map, the horizontal axis represents distance along the colon (left to right $=$ proximal to distal; only the last $7.27 \mathrm{~cm}$ proximal to the anus were evaluated, each horizontal square represents a 10th of this distance) whereas the vertical axis represents time of recording (top to bottom $=0$ to 120 seconds, each vertical square represents 3 seconds). Instant position of fecal pellets is represented as black squares in each cell of the STM; movement is shown as a horizontal change of the fecal pellet position. PS: propulsion speed of fecal pellets, measured directly from the fluoroscopic recording, where D represents distance travelled by the fecal pellets (the red arrows in frames 1 and 120 show the position of the pellet at the beginning of the recording; the green arrow in frame 120 represents the position of the pellet at the end of the recording) and $\mathrm{T}$, duration of the recording analyzed. (B) Analysis of the STM, MIT: maximum period during which the fecal pellet did not move (maximum immobility time, in seconds), FP exits: time at which the fecal pellet eventually exited the colon, PS': propulsion speed measured from the STM, where D' is distance travelled by the fecal pellets (in $\mathrm{cm}$ ) and $\mathrm{T}$, duration of the recording analyzed (in seconds).
As shown in Table, the $\%$ of videos that could be analyzed was $73-100 \%$ for all experimental groups (only 24/154 movies, were discarded, generally due to excessive animal movement, $15.6 \%$ ). Most analyzed movies were 120 seconds long (only 18/130 movies were shorter, $13.8 \%$ ) and had stained fecal pellets in the colon at the time of recording (only 9/130 did not, 6.9\%). At least 6 movies per experimental group were included for further analysis $(n=7-14$, 6-8, and 7-10 for the MOR, LOP, and WIN sets of experiments, 
Table. Basic Features of Movies Used for the Analyses

\begin{tabular}{|c|c|c|c|c|c|c|c|}
\hline \multicolumn{2}{|c|}{ Experimental groups } & \multirow{2}{*}{$\begin{array}{l}\mathrm{N} \\
16\end{array}$} & \multirow[t]{2}{*}{$\mathrm{A}(\%)$} & \multirow[t]{2}{*}{$\mathrm{D}(\mathrm{sec})$} & \multirow{2}{*}{$\frac{\mathrm{B}(\%)}{13(100.0)}$} & \multirow{2}{*}{$\begin{array}{c}\text { MFP } \\
4.08 \pm 0.70\end{array}$} & \multirow{2}{*}{$\begin{array}{l}\text { Diam }(\mathrm{mm}) \\
4.87 \pm 0.21\end{array}$} \\
\hline MOR & Saline & & & & & & \\
\hline & & & $1: 40 \mathrm{sec} ; 1: 66 \mathrm{sec} ; 1: 50 \mathrm{sec}$ & 1:87 sec; $1: 93 \mathrm{sec}$ & & & \\
\hline & MOR 0.1 & 16 & $14(87.5)$ & $118 \pm 1$ & $14(100.0)$ & $2.14 \pm 0.26$ & $5.29 \pm 0.20$ \\
\hline & & & $2: 0 \mathrm{sec}$ & $1: 95 \mathrm{sec}$ & & & \\
\hline & MOR 1 & 12 & $11(91.7)$ & $120 \pm 0$ & $10(91.7)$ & $3.80 \pm 0.72$ & $4.71 \pm 0.22$ \\
\hline & & & $1: 0 \mathrm{sec}$ & & & & \\
\hline & MOR 5 & 8 & $8(100.0)$ & $120 \pm 0$ & $7(87.5)$ & $1.86 \pm 0.23$ & $5.34 \pm 0.20$ \\
\hline & MOR 10 & 12 & $10(83.3)$ & $120 \pm 0$ & $10(100.0)$ & $2.70 \pm 0.80$ & $4.97 \pm 0.19$ \\
\hline & & & $1: 44 \mathrm{sec} ; 1: 0 \mathrm{sec}$ & & & & \\
\hline \multirow[t]{9}{*}{ LOP } & CREM & 12 & $8(66.7)$ & $119 \pm 1$ & $7(87.5)$ & $3.71 \pm 2.32$ & $5.29 \pm 0.23$ \\
\hline & & & $1: 43 \mathrm{sec} ; 1: 60 \mathrm{sec} ; 2: 0 \mathrm{sec}$ & $1: 110 \mathrm{sec}$ & & & \\
\hline & LOP 0.1 & 12 & $10(83.3)$ & $116 \pm 3$ & $8(80.0)$ & $3.00 \pm 0.40$ & $4.65 \pm 0.16$ \\
\hline & & & $1: 20 \mathrm{sec} ; 1: 0 \mathrm{sec}$ & 1:97 sec; $1: 99 \mathrm{sec}$ & & & \\
\hline & LOP 1 & 11 & $8(72.7)$ & $114 \pm 4$ & $6(75.0)$ & $2.83 \pm 0.66$ & $4.94 \pm 0.21$ \\
\hline & & & $1: 54 \mathrm{sec} ; 1: 60 \mathrm{sec} ; 1: 0 \mathrm{sec}$ & $1: 101 \mathrm{sec} ; 1: 88 \mathrm{sec}$ & & & \\
\hline & LOP 5 & 8 & $8(100.0)$ & $120 \pm 0$ & $8(100.0)$ & $2.75 \pm 0.44$ & $4.66 \pm 0.18$ \\
\hline & LOP 10 & 8 & $6(75.0)$ & $117 \pm 3$ & $6(100.0)$ & $3.00 \pm 0.89$ & $4.82 \pm 0.19$ \\
\hline & & & $1: 60 \mathrm{sec} ; 1: 62 \mathrm{sec}$ & $1: 101 \mathrm{sec}$ & & & \\
\hline \multirow[t]{8}{*}{ WIN } & TOCRI & 12 & $9(75.0)$ & $106 \pm 3$ & $7(77.77)$ & $3.67 \pm 0.73$ & $4.69 \pm 0.26$ \\
\hline & & & $1: 53 \mathrm{sec} ; 2: 0 \mathrm{sec}$ & $1: 79 \mathrm{sec} ; 1: 77 \mathrm{sec} ; 1: 82 \mathrm{sec}$ & & & \\
\hline & WIN 0.1 & 12 & $10(83.3)$ & $111 \pm 4$ & $10(100.0)$ & $3.80 \pm 0.47$ & $4.76 \pm 0.13$ \\
\hline & & & $2: 0 \mathrm{sec}$ & $1: 86 \mathrm{sec} ; 1: 85 \mathrm{sec} ; 1: 107 \mathrm{sec}$ & & & \\
\hline & & & & $1: 113 \mathrm{sec}$ & & & \\
\hline & WIN 1 & 8 & $8(100.0)$ & $112 \pm 5$ & $8(100.0)$ & $4.38 \pm 0.35$ & $4.61 \pm 0.15$ \\
\hline & & & & 1:81 sec; $1: 93 \mathrm{sec}$ & & & \\
\hline & WIN 5 & 7 & $7(100.0)$ & $120 \pm 0$ & $7(100.0)$ & $2.57 \pm 0.40$ & $4.56 \pm 0.25$ \\
\hline
\end{tabular}

$\mathrm{N}$, number of animals included in the study per experimental group and time point; A (\%), number and \% of movies that were used for further analyses (movies in which rat movements did not allow for the analyses to be performed for longer than 75 seconds were excluded; their duration is shown in italics, second and further rows in each cell; D, mean duration of movie segments used for further analyses (obtained from the movies included in A, given in seconds): most recordings were used for their whole duration (120 seconds); duration of shorter useful movie segments is shown in italics; B (\%), number and \% of analyzed movies (obtained from A) in which animals had stained fecal pellets in their colon at the time of recording and from which spatio-temporal maps (STM) where built; MFP, mean number of stained fecal pellets per STM; Diam, diameter of each fecal bolus displayed on the fluoroscopic recording was measured with Image J; MOR, animals received morphine at 0.1 (MOR 0.1), 1 (MOR 1), 5 (MOR 5) or $10 \mathrm{mg} / \mathrm{kg}$ (MOR 10) intraperitoneally (ip); CREM, animals received Cremophor 20\% in saline, at $1 \mathrm{~mL} /$ kg; LOP, animals received loperamide at 0.1 (LOP 0.1), 1 (LOP 1), 5 (LOP 5) or $10 \mathrm{mg} / \mathrm{kg}$ (LOP 10); TOCRI, animals received Tocrisolve at $1 \mathrm{~mL} / \mathrm{kg}$; WIN, animals received WIN 55,212-2 at 0.1 (WIN 0.1), 1 (WIN 1) or $5 \mathrm{mg} / \mathrm{kg}$ (WIN 5).

Rats were injected ip with different substances as MOR, CREM, LOP, TOCRI, and WIN. For each session, rats were gavaged a load of contrast medium (barium sulfate, $2 \mathrm{~g} / \mathrm{mL}, 1.5 \mathrm{~mL}$ ) at least 20 hours before, so that stained fecal pellets could be found within the colon at the time of recording.

respectively). Thus, $78.6 \%$ (121/154) of movies were at least 75 seconds long and had at least one stained fecal pellet in the colon. STM were built from these recordings (see representative STM in Fig. 3).

Compared to control animals, no significant differences were found in morphine, loperamide, and WIN 55,212-2-treated rats (irrespective of the dose) for the MFP per STM and their mean diameter (Diam) $(P>0.05$, one-way ANOVA; Table).

Since different vehicles (saline for morphine, Cremophor for loperamide, and Tocrisolve for WIN 55,212-2) were used in the 3 different sets of experiments, we compared their effect on the dif- ferent parameters of interest first (Fig. 4). Tocrisolve significantly increased PS and PS', and both Cremophor and Tocrisolve significantly reduced MIT. Compared to saline-treated animals, in Cremophor/Tocrisolve animals, the percentage of fast pellets tended to increase, and the percentage of fast STM significantly increased, but no significant difference in the percentage of STM with fecal pellets exiting the colon was found. Thus, both vehicles, particularly Tocrisolve, tended to increase the colonic propulsive activity of endogenous fecal pellets.

We describe next the results obtained for each particular drug. 
A

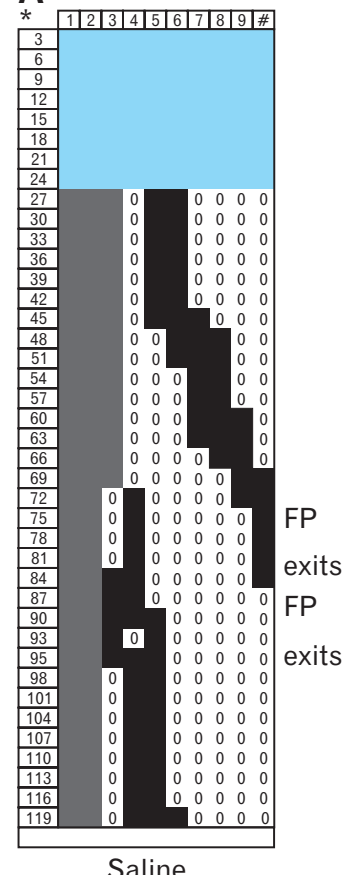

Saline

B

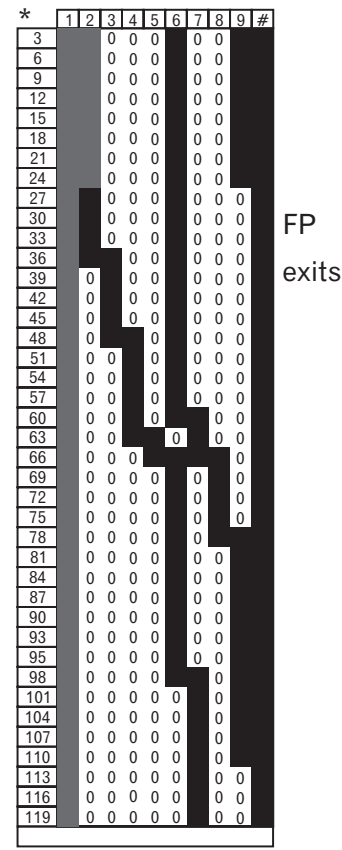

CREM

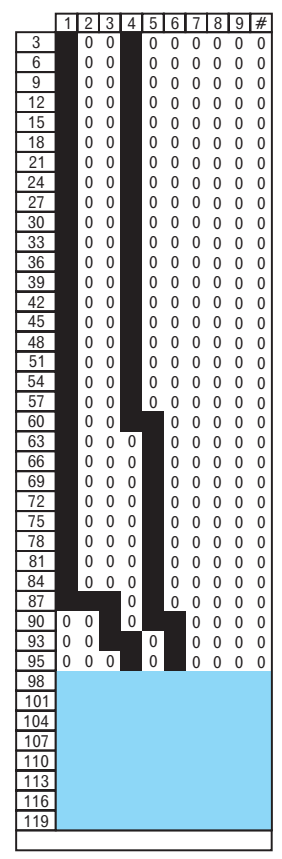

MOR 0.1

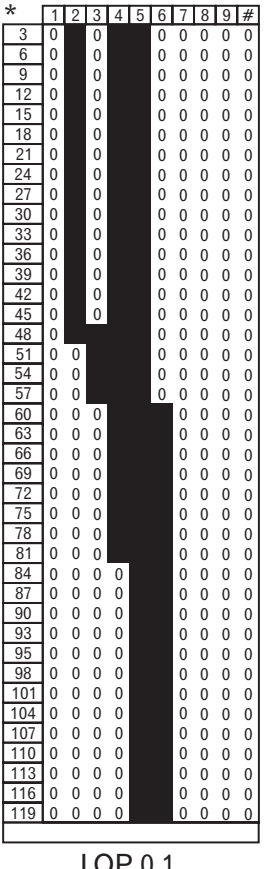

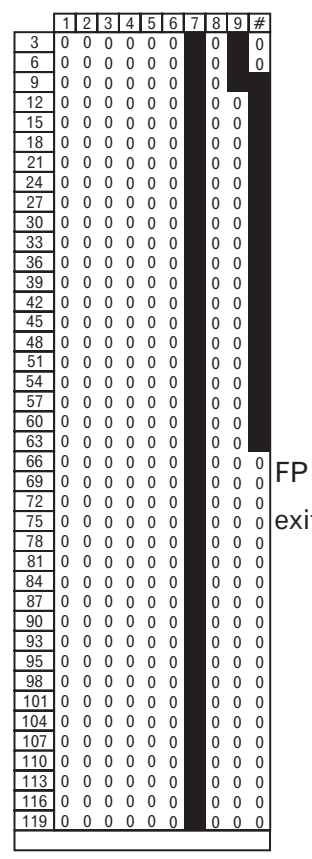

MOR 5

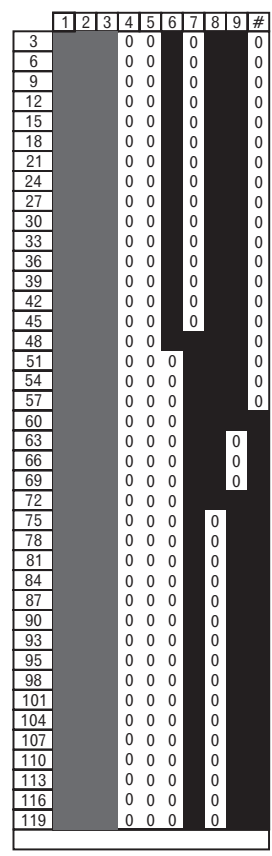

LOP 5

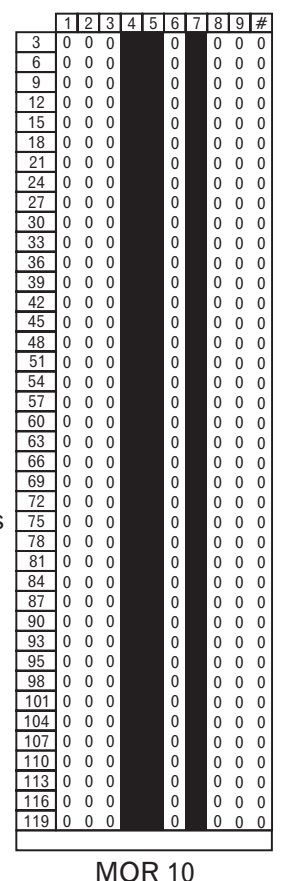

MOR 10

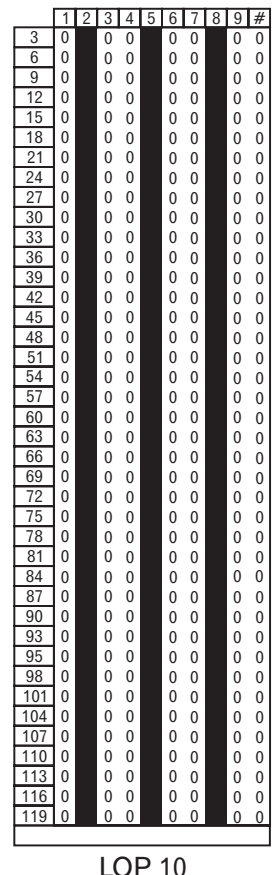

Figure 3. Effects of morphine, loperamide, and WIN 55,212-2: representative examples. (A) Representative spatio-temporal maps (STM) for salineand morphine-treated rats at different doses (MOR; 0.1, 5, and $10 \mathrm{mg} / \mathrm{kg}$ ). (B) Representative STM for Cremophor-treated (CREM) and loperamidetreated rats at different doses (LOP; 0.1, 5 , and $10 \mathrm{mg} / \mathrm{kg}$ ). (C) Representative STM for Tocrisolve-treated (TOCRI) and WIN 55,212-2-treated rats at different doses (WIN; 0.1, 1, and $5 \mathrm{mg}$ / $\mathrm{kg})$. In each STM, the horizontal axis corresponds to distance $(7.27 \mathrm{~cm})$ and the vertical axis corresponds to time (120 seconds). Grey rectangles represent parts of the recordings in which fecal pellets could not be seen due to overlapping of other gastrointestinal structures; blue rectangles represent parts of recordings that could not be analyzed due to sudden movement of the animal. See legend for Figure 2 for more details.

\section{Effects of Morphine}

Figure $3 \mathrm{~A}$ shows representative STM for rats treated with saline and morphine at different doses. Quantitative results of the analysis performed for colonic motor activity in these animals are shown in Figure 5.
Compared to saline, morphine at $0.1 \mathrm{mg} / \mathrm{kg}$ tended to increase the PS, but the difference was not statistically different. However, PS decreased for all the remaining MOR doses and, compared to MOR 0.1, the difference was statistically significant for MOR 1 and MOR 10 (Fig. 5A). A similar result was found when the PS was calculated from the STM (PS') (Supplementary Fig. 2A). 


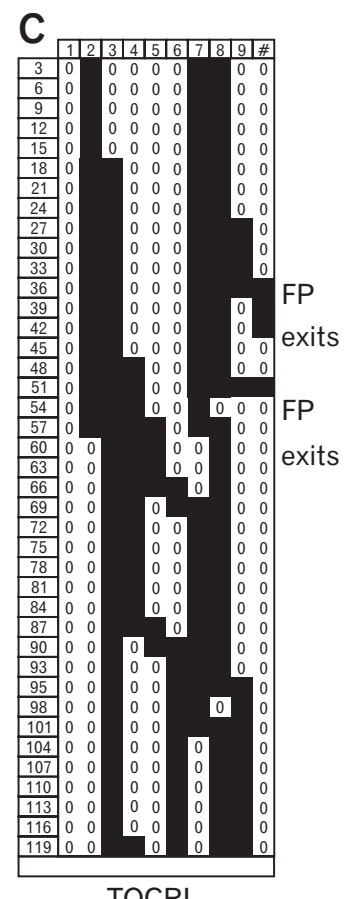

TOCRI

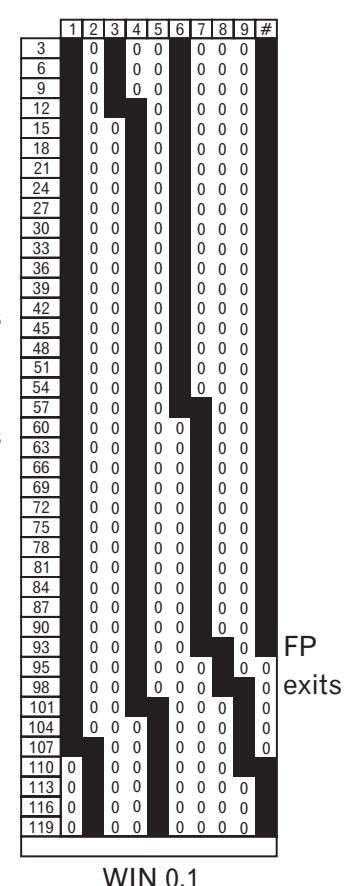

WIN 0.1

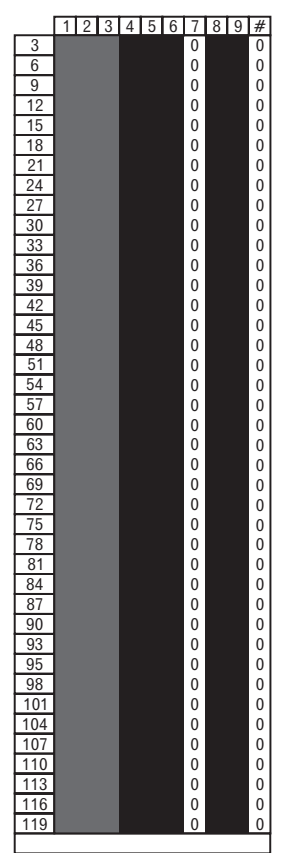

WIN 1

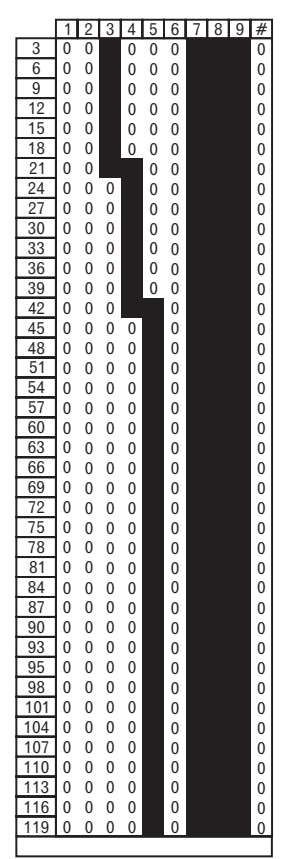

WIN 5

Figure 3. Continued.

MIT was significantly increased for morphine at $10 \mathrm{mg} / \mathrm{kg}$ compared to saline, MOR 0.1, and MOR 1 (but not MOR 5) groups, and reduced for MOR 0.1 compared to saline, although this difference did not reach statistical significance (Fig. 5B).

Interestingly, the $\%$ of slow and fast pellets increased and decreased, respectively, in a dose-dependent manner $\left(P=0.001, \chi^{2}\right.$ test) (Fig. 5C). Similar results were found for the analysis of the percentage of slow and fast STM according to the speed of their pellets (Group 1: all the fecal pellets in the map moved 0-2.5 cm; Group 2: at least one fecal pellet in the map moved $\geq 2.5 \mathrm{~cm})(P$ $=0.021, \chi^{2}$ test (Fig. 5D). However, in agreement with the results shown for PS and MIT, animals treated with MOR 0.1 showed a higher percentage of STM with fast pellets (Group 2). Finally, the percentage of STM with fecal pellets that exited the colon during the recording decreased with the dose of morphine and the difference was close to statistical significance $\left(P=0.057, \chi^{2}\right.$ test). This was particularly clear in the case of animals treated with morphine at the highest dose $(10 \mathrm{mg} / \mathrm{kg})$, for which no fecal pellet exited the colon (Fig. 5E).

\section{Effects of Loperamide}

Figure 3B shows representative STM for rats treated with Cremophor and loperamide at different doses. Quantitative results of the analysis performed for colon motor activity in these animals are shown in Figure 6.
As for morphine, PS was slower in the LOP 5 and LOP 10 groups compared to Cremophor, and LOP 0.1 and LOP 1 groups (Fig. 6A), although the difference did not reach statistical significance. A similar result was found when the propulsion speed was calculated from the STM (PS'), but in this case, the difference was statistically significant for LOP 10 group vs control group (Cremophor) (Supplementary Fig. 2B). In addition, there was a clear dose-dependent increase in MIT, which was significantly higher for loperamide at $5 \mathrm{mg} / \mathrm{kg}$ and $10 \mathrm{mg} / \mathrm{kg}$ compared to Cremophor and LOP $0.1 \mathrm{mg} / \mathrm{kg}$ groups (Fig. 6B).

As for morphine too, the percentage of slow and fast pellets increased and decreased, respectively, in a dose-dependent manner ( $P$ $=0.00001, \chi^{2}$ test) (Fig. 6C). When we classified STM according to the speed of their pellets in the 2 categories mentioned above, the percentage of maps with slow pellets increased in a significant manner ( $P=0.0011, \chi^{2}$ test $)$ (Fig. 6D). Finally, the percentage of STM with fecal pellets that exited the colon during the recording decreased with the dose of loperamide in a statistically significant manner $\left(P=0.029, \chi^{2}\right.$ test $)$. This was particularly clear in the case of animals treated with loperamide at the highest doses $(5 \mathrm{mg} / \mathrm{kg}$ and $10 \mathrm{mg} / \mathrm{kg}$ ) (Fig. 6E).

\section{Effects of WIN 55,212-2}

Figure 3C shows representative STM for rats treated with Tocrisolve and WIN 55,212-2 at different doses. Quantitative re- 

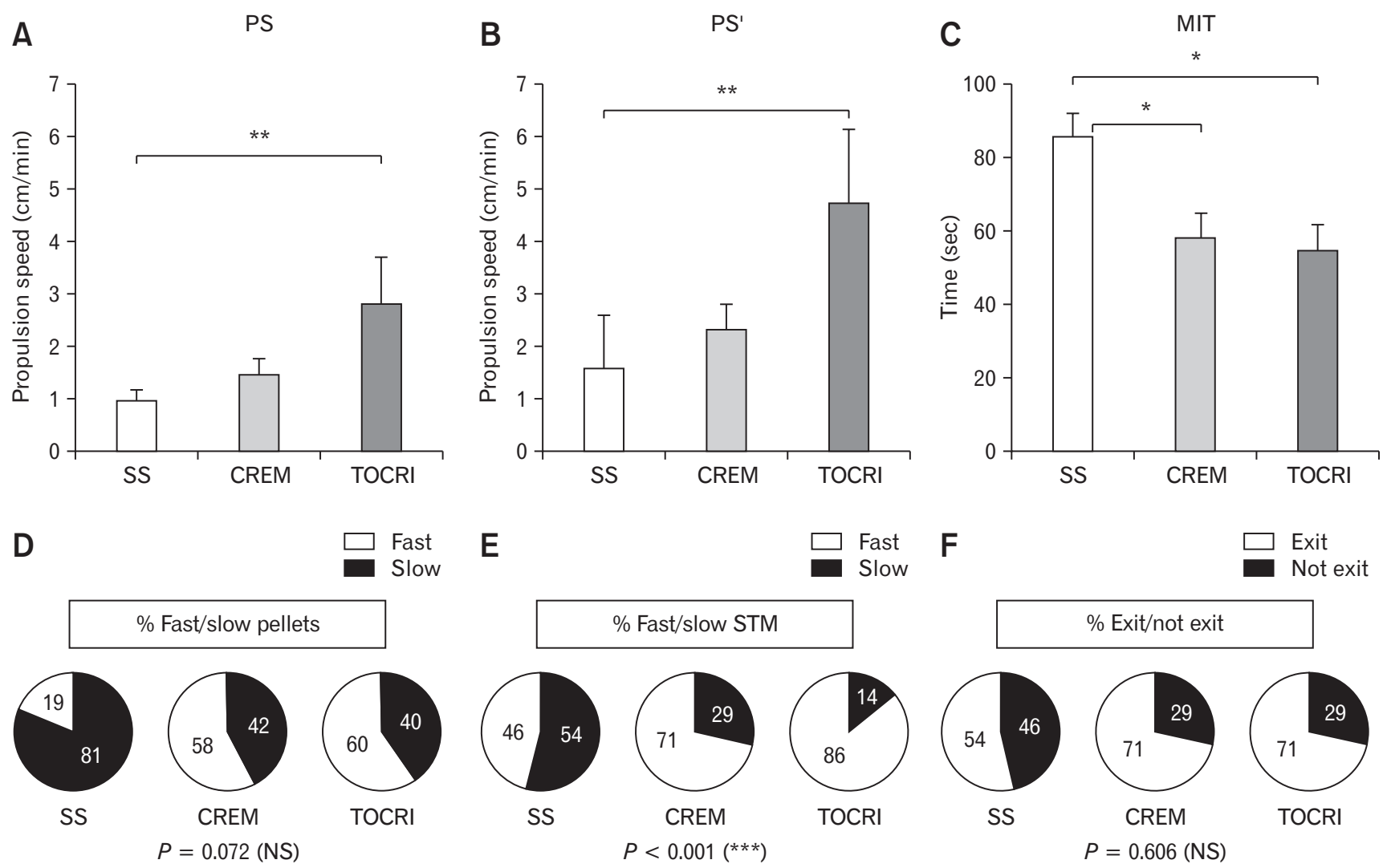

Figure 4. Effects of vehicles: quantitative analysis. (A-C) Bar graphs for (A) fecal pellet propulsion speed (PS), (B) fecal pellet propulsion speed measured from the spatio-temporal maps (STM; PS'), (C) maximum immobility time (MIT). Results are shown as the mean \pm SEM. ${ }^{*}<0.05$; ${ }^{* *} P<0.01$ (one-way ANOVA followed by post-hoc Bonferroni multiple comparison test). (D-F) Pie charts displaying: (D) the distribution of fecal pellets moving slowly $(0-2.5 \mathrm{~cm}$ : black, slow pellets) and quickly ( $\geq 2.5 \mathrm{~cm}$ : white, fast pellets) as shown in the STM during the fluoroscopy sessions; (E) the distribution of STM as "slow" (all pellets moved 0-2.5 cm: black) and "fast" (at least one pellet moved $\geq 2.5 \mathrm{~cm}$ : white); (F) the distribution of STM showing fecal pellets that exited the colon (white) or not (black). The results of the statistical analysis are shown at the foot of the pie charts. ${ }^{* *} P<0.001$ ( $\chi^{2}$ test). Animals received vehicles at $1 \mathrm{~mL} / \mathrm{kg}$ by intraperitoneal (ip) injection. $\mathrm{NS}$, not significant; SS, saline (NaCl $0.9 \%$ in distilled water); CREM, Cremophor (20\% in saline); TOCRI, Tocrisolve.

sults of the analysis performed for colon propulsive activity in these animals are shown in Figure 7.

PS was significantly lower in animals treated with WIN at 1 $\mathrm{mg} / \mathrm{kg}$ and $5 \mathrm{mg} / \mathrm{kg}$, compared to Tocrisolve and WIN 0.1 groups (Fig. 7A). A similar result was found when the propulsion speed was calculated from the STM (Supplementary Fig. 2C). Again, MIT tended to increase for WIN at $1 \mathrm{mg} / \mathrm{kg}$ and $5 \mathrm{mg} / \mathrm{kg}$ compared to Tocrisolve and WIN 0.1, although the difference did not reach statistical significance (Fig. 7B).

The percentage of slow and fast pellets increased and decreased, respectively, in a dose-dependent manner $\left(P=0.020, \chi^{2}\right.$ test) (Fig. 7C). When we classified STM according to the speed of their pellets in the two categories mentioned above, the percentage of maps with slow and fast pellets tended to increase and decrease, respec- tively, in a dose-dependent manner, but the difference did not reach statistical significance $\left(P=0.101, \chi^{2}\right.$ test $)$ (Fig. 7D). Finally, the percentage of STM with fecal pellets that exited the colon during the recording decreased with the dose of WIN 55,212-2, but the difference did not reach statistical significance $\left(P=0.096, \chi^{2}\right.$ test $)$ (Fig. 7E). Thus, the WIN group showed a very similar tendency to MOR and LOP groups.

\section{Discussion}

Here we used fluoroscopic recordings and STM to evaluate, in conscious rats, the alterations of colonic propulsive activity associated to the acute administration of increasing doses of analgesics known to inhibit colonic motility. 


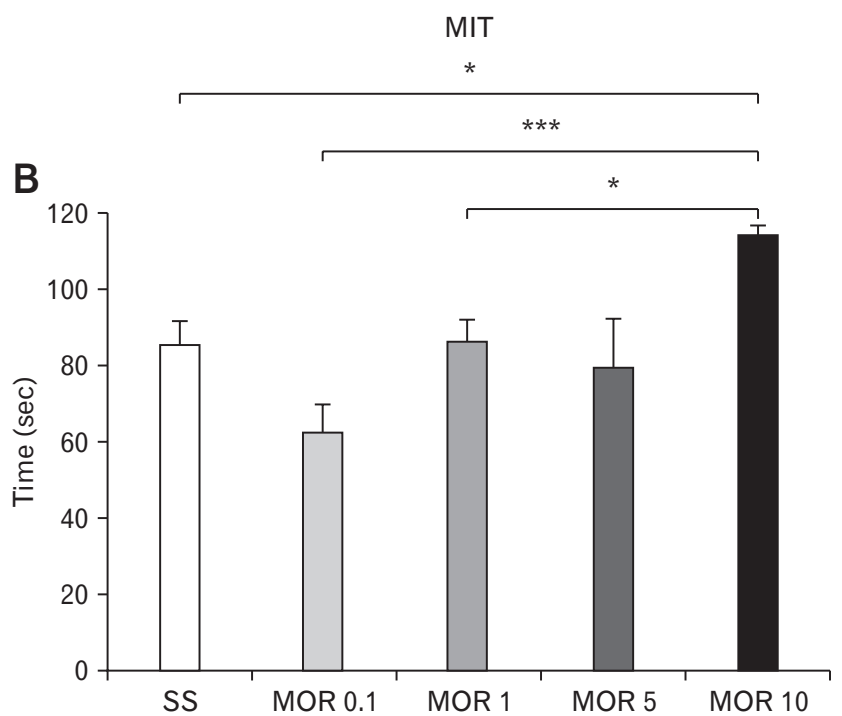

C

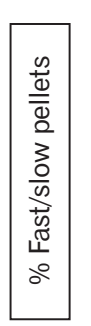

D

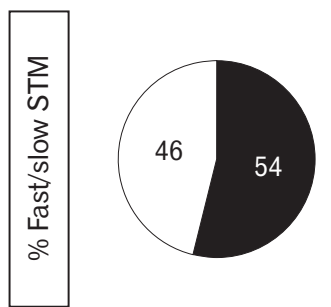

E

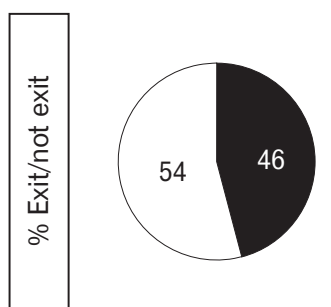

MOR 0.1
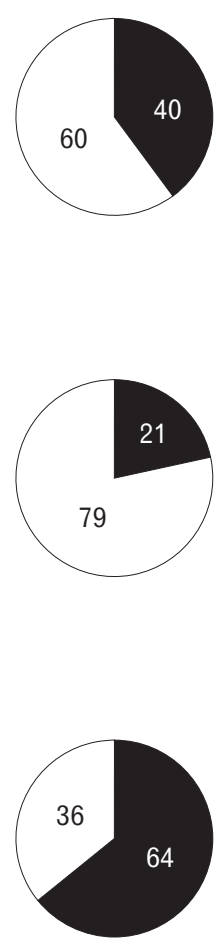
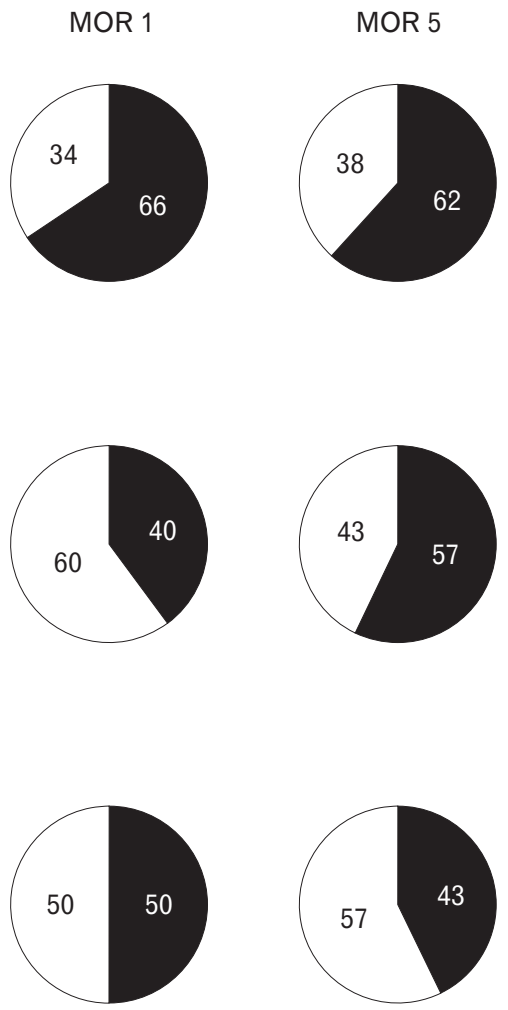

MOR 10
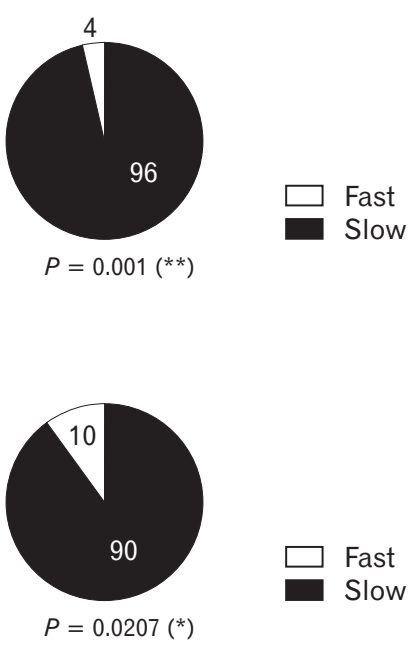

Exit

Not exit

Figure 5. Effects of morphine: quantitative analysis. (A-B) Bar graphs for (A) propulsion speed (PS); (B) maximum immobility time (MIT). Results are shown as the mean \pm SEM. ${ }^{*}<<0.05 ;{ }^{* *} P<0.001$ (one-way ANOVA followed by post-hoc Bonferroni multiple comparison test). (C-E) Pie charts displaying: (C) the distribution of fecal pellets moving slowly $(0-2.5 \mathrm{~cm}$ : black, slow pellets) and quickly ( $\geq 2.5 \mathrm{~cm}$ : white, fast pellets) as shown in the spatio-temporal maps (STM) during the fluoroscopy sessions; (D) the distribution of STM as "slow" (all pellets moved 0-2.5 cm: black) and "fast" (at least one pellet moved $\geq 2.5 \mathrm{~cm}$ : white); (E) the distribution of STMs showing fecal pellets that exited (white) or not (black) the colon. The results of the statistical analysis are shown at the foot of the pie charts. ${ }^{*} P<0.05$, **P<0 0.01 ( $\chi^{2}$ test). NS, not significant; SS, animals received saline $(\mathrm{NaCl} 0.9 \%$ in distilled water, at $1 \mathrm{~mL} / \mathrm{kg}$ intraperitoneally [ip]); MOR, animals received morphine at $0.1,1,5$ or $10 \mathrm{mg} / \mathrm{kg}$ (ip). 


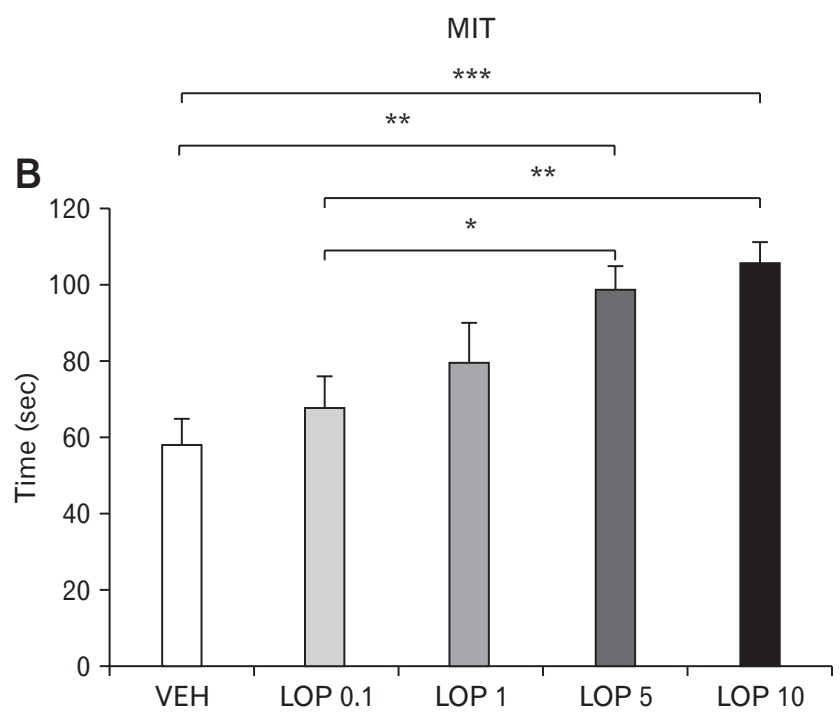

C

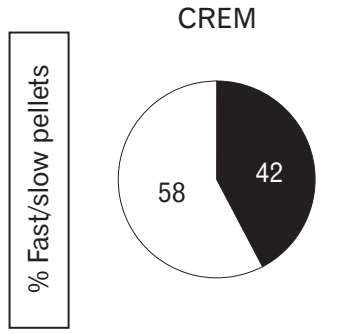

D

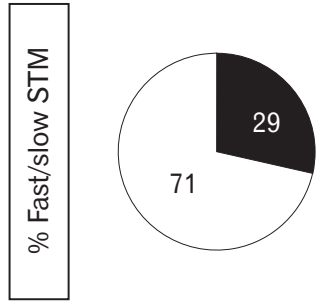

E

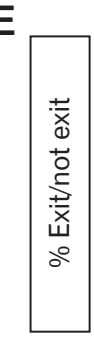

LOP 0.1
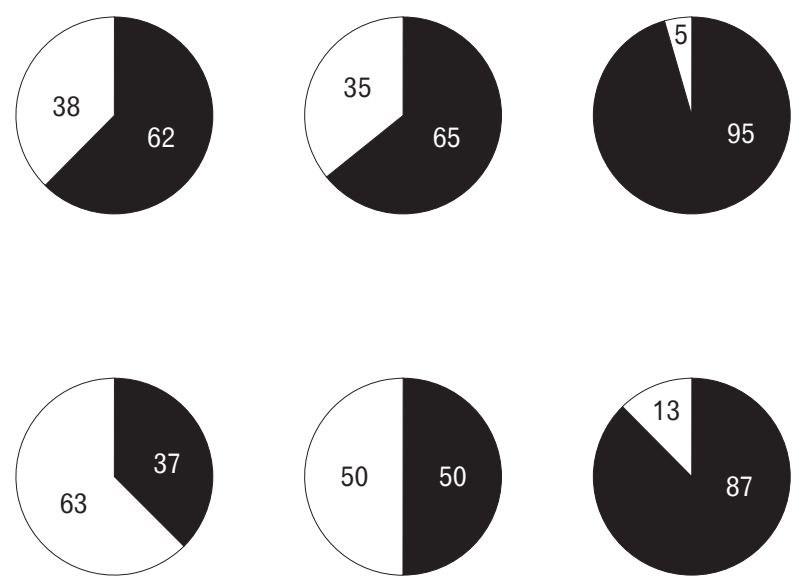

95

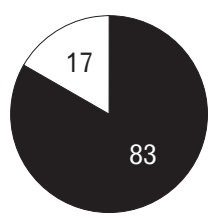

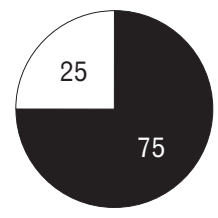

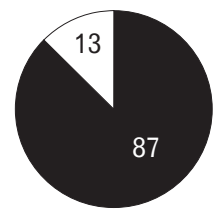

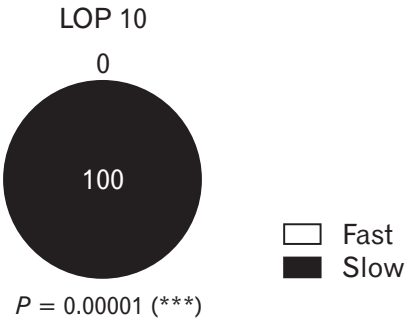
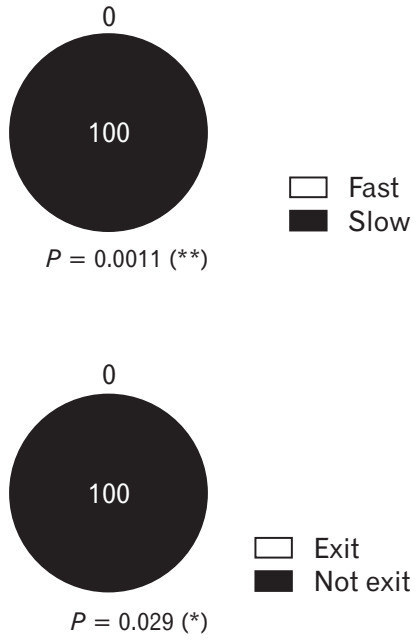

Figure 6. Effects of loperamide: quantitative analysis. (A, B) Bar graphs for (A) propulsion speed (PS); (B) maximum immobility time (MIT). Results are shown as the mean \pm SEM. ${ }^{*} P<0.05 ;{ }^{*} P<0.01 ;{ }^{* *} P<0.001$ (one-way ANOVA followed by post-hoc Bonferroni multiple comparison test). (C-E) Pie charts displaying: (C) the distribution of fecal pellets moving slowly (0-2.5 cm: black, slow pellets) and quickly ( $\geq$ $2.5 \mathrm{~cm}$ : white, fast pellets) as shown in the spatio-temporal maps (STM) during the fluoroscopy sessions; (D) the distribution of STM as "slow" (all pellets moved $0-2.5 \mathrm{~cm}$ : black) and "fast" (at least one pellet moved $\geq 2.5 \mathrm{~cm}$ : white); (E) the distribution of STM showing fecal pellets that exited (white) or not (black) the colon. The results of the statistical analysis are shown at the foot of pie charts. ${ }^{*} P<0.05 ;{ }^{* *} P<0.01 ;{ }^{* *} P<0.001$ ( $\chi^{2}$ test). CREM, animals received Cremophor $20 \%$ in saline, at $1 \mathrm{~mL} / \mathrm{kg}$ intraperitoneally (ip); LOP, animals received loperamide at $0.1,1,5$ or $10 \mathrm{mg} / \mathrm{kg}$ (ip). 

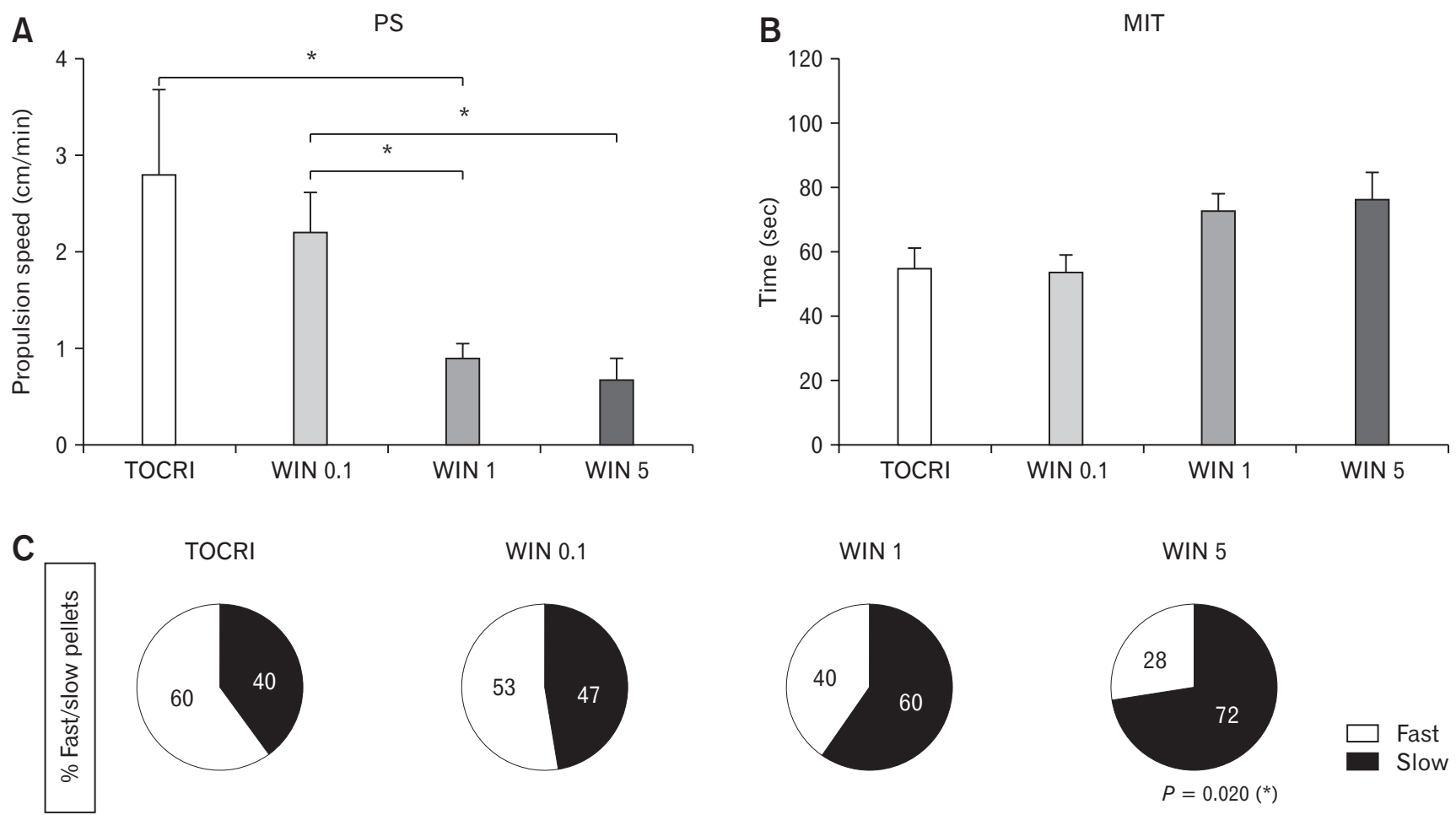

WIN 0.1

WIN 1

WIN 5
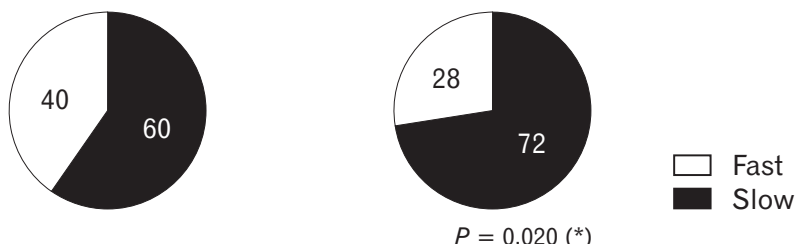

D
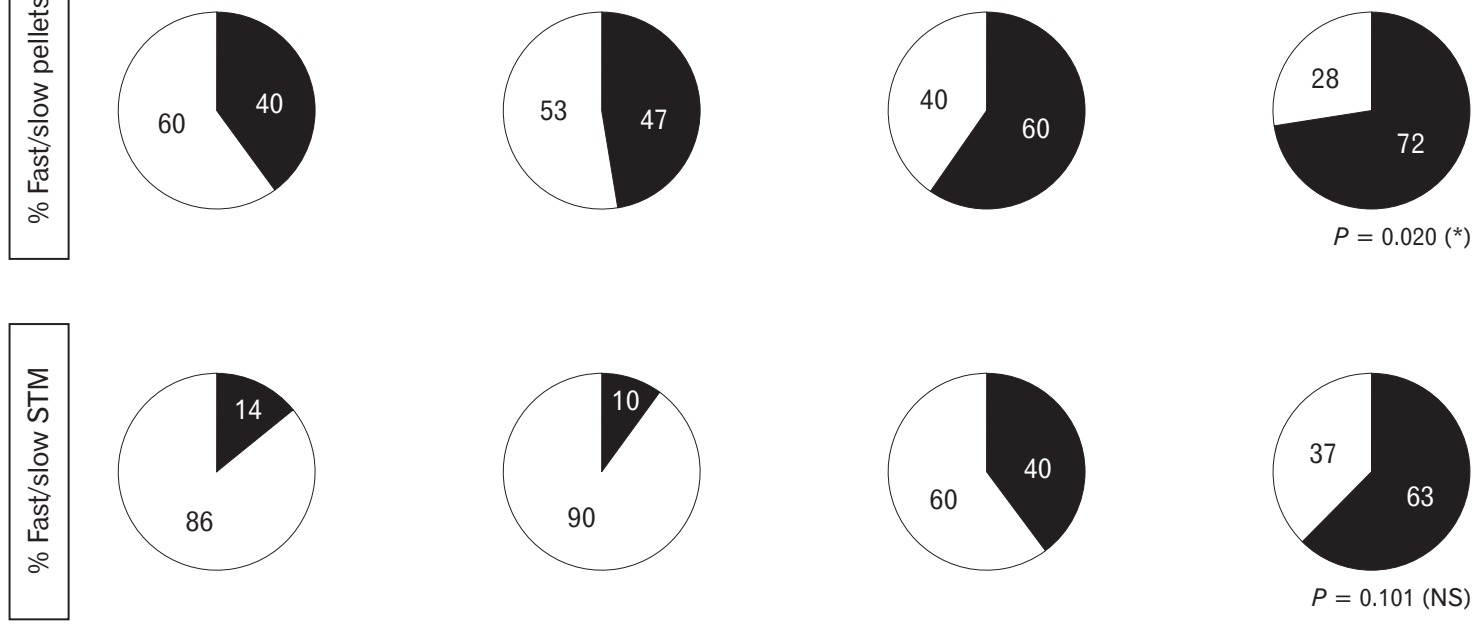

Fast

E
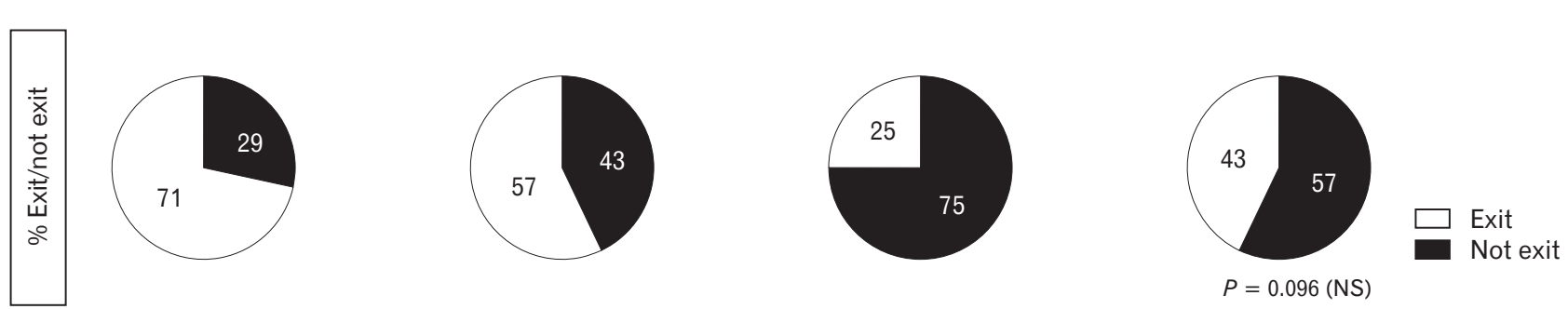

Figure 7. Effects of WIN 55,212-2: quantitative analysis. (A, B) Bar graphs for (A) propulsion speed (PS); (B) maximum immobility time (MIT). Results are shown as the mean \pm SEM. ${ }^{*} P<0.05$ (one-way ANOVA followed by post-hoc Bonferroni multiple comparison test). (C) The distribution of fecal pellets moving slowly $(0-2.5 \mathrm{~cm}$ : black, slow pellets) and quickly ( $\geq 2.5 \mathrm{~cm}$ : white, fast pellets) as shown in the STM during the fluoroscopy sessions; (D) the distribution of spatio-temporal maps (STM) as "slow" (all pellets moved 0-2.5 cm: black) and "fast" (at least one pellet moved $\geq 2.5 \mathrm{~cm}$ : white); (E) the distribution of STMs showing fecal pellets that exited (white) or not (black) the colon. The results of the statistical analysis are shown at the foot of pie charts. ${ }^{*}<0.05$ ( $\chi^{2}$ test). NS, not significant; TOCRI, animals received Tocrisolve $7 \%$ in saline, at $1 \mathrm{~mL} / \mathrm{kg}$ intraperitoneally (ip); WIN, animals received WIN 55,212-2 at $0.1,1$ or $5 \mathrm{mg} / \mathrm{kg}$ (ip). 
Many techniques have been used so far to study motility of the gastrointestinal tract in humans and experimental animals. ${ }^{9,26,27}$ Specifically, radiographic methods have been used from the end of the 19th century until now. ${ }^{28}$ Among other imaging methods, magnetic resonance has been postulated as the "gold standard" method to non-invasively study the intestinal motor alterations in humans, and it offers both functional and morphologic data. ${ }^{29}$ But its application to experimental animals, particularly rodents, like many other techniques, requires the use of anesthesia, which may interfere with intestinal motility. ${ }^{7,8}$ Fluoroscopy (continuous radiographic recording), which also offers functional and morphologic data, may be a good alternative to study intestinal motility in vivo in conscious animals.

Research using fluoroscopy without anesthetics has been previously performed in humans, ${ }^{30,31}$ and in medium-big sized animals like rabbits, ${ }^{32}$ sheep ${ }^{33}$ and pigs. ${ }^{34}$ In contrast, anesthetics were generally used in fluoroscopy recordings obtained from mice. ${ }^{35}$ Recently, we applied fluoroscopy in conscious rats to study the motor function of small intestine in control animals ${ }^{15}$ and the colonic propulsive activity of endogenous fecal pellets after vincristine administration. ${ }^{16}$ Here, we applied fluoroscopy to study, also in non-anaesthetized rats, the acute effect of opioid and cannabinoid agonists (known to produce analgesia and to inhibit gastrointestinal motility) on colonic propulsive activity.

STM have been used for a long time in organ bath experiments to analyze motor patterns of gut segments in response to fluid infusion $^{12,36-39}$ as well as colonic fecal pellet propulsion..$^{13,40,41}$ They have also been applied to recordings obtained in vivo from anesthetized rats, using magnetic resonance ${ }^{42}$ and from conscious mice using fluoroscopy to study gastric and duodenal motor activity. ${ }^{43,44}$ In our studies, STM were built from fluoroscopic recordings obtained from conscious rats. Whereas STM were easily built automatically from fluoroscopic recordings to represent motor function of wellstained small intestinal segments, based on the changes in diameter along time and segment position, ${ }^{15}$ the faint staining of many fecal pellets (that could be, nevertheless, followed by eye), did not allow to apply automatic procedures. Therefore, STM were manually built in this and our previous study to represent fecal pellet position along the colon. ${ }^{16}$ Only the last $7.27 \mathrm{~cm}$ proximal to the anus were evaluated. This was the length of the distal colon that could be measured in all rats, a part of the colon that in this species runs straight and parallel to the spine. This part of the colon was generally free of interference with the stained cecum (shown as grey areas in the representative STM; Fig. 3). Compared with in vitro studies, in which most experiments are performed after inserting a single fecal pellet through the oral end of the emptied colon, one or more stained endogenous fecal pellets could be found in each rat during fluoroscopy. Since the recordings had a similar duration (Table), we used the terms "fast" and "slow" to categorize the pellets depending on whether they transited more or less length, respectively, with an arbitrary cut distance of $2.5 \mathrm{~cm}$ (approximately a third of the whole distance evaluated). STM were used to "summarize" the effect of the drug in each rat. Thus, for a particular STM, if no pellet could move $2.5 \mathrm{~cm}$ or more, the STM was considered slow (all its fecal pellets were slow). On the contrary, if at least one fecal pellet of a particular STM could move $2.5 \mathrm{~cm}$ or more, the STM was considered fast. Although based on an arbitrary categorization, the results obtained with the STM approach were consistent with the remaining analyses and therefore we consider this method useful to represent drug-induced changes in endogenous fecal pellet movement from fluoroscopy recordings.

In the present study, we evaluated 3 analgesic drugs (morphine, loperamide, and WIN 55,212-2), known to suppress gastrointestinal motor function. In previous studies using radiographic techniques, all these drugs produced a dose-dependent inhibition of general gastrointestinal motility ${ }^{9,10}$ (non-published observations for loperamide). However, in those experiments, barium was administered just after the drug. Therefore, the effects of the drugs on more proximal parts of the gastrointestinal tract (stomach, small intestine, and cecum) delayed barium staining of fecal pellets to late timepoints in the experiment and very little could be deduced on the direct effects of the drugs on colon motor function because at the time fecal pellets could be seen, the effect of the drug could already be residual or inexistent. The direct effect of a drug on colonic propulsion of endogenous fecal pellet movement can more reliably be analyzed if the drug is given when fecal pellets are already stained or about to be stained, like in the present study. In the present study, fecal pellets found in the colon were already stained by the barium administered 20-22 hours before the experiment, and thus the effects of the drugs on colonic fecal pellet propulsion could be directly visualized.

The rats were of similar age and weight and drugs (or their vehicles) did not induce any significant modification in fecal pellet length (not shown) or diameter, probably because they were administered just 30 minutes before the corresponding fluoroscopic recordings. Maybe longer exposure time to these drugs might modify these parameters. For example, fecal pellets from mice repeatedly treated with the antineoplastic drug irinotecan showed statistically significant differences in length along treatment. ${ }^{45}$ Likewise, vincristine, another antitumoral drug, reduced the diameter of fecal pellets 
in the rat, when repeatedly administered. ${ }^{16}$ Whatever the case may be, the fact that morphometric values of the endogenous fecal pellets were not significantly changed in our experimental conditions imply that changes in fecal pellet propulsion did not involve altered mechanical stimulation of the colon associated to changes in fecal pellet size.

Ideally, vehicles should not exert any effect. Compared to animals treated with saline (control for morphine), animals that received the vehicles for loperamide (Cremophor) and WIN 55,2122 (Tocrisolve) showed faster propulsive activity of endogenous fecal pellets. Cremophor and Tocrisolve are vehicles commonly used for in vitro and in vivo experiments when lipid-soluble drugs are to be used. Interestingly, these vehicles did not affect nociceptive thresholds. ${ }^{20,21,46}$ This suggests that gastrointestinal motor function might be more sensitive to these particular vehicles. Studies on gastrointestinal motility are very scarce, but in one of them, this function was not altered by Cremophor RH 40 (a derivative of castor oil, different from Cremophor EL, which was used here). ${ }^{47}$ Thus, more research on the effects of vehicles for non-water-soluble drugs on gastrointestinal motility and other functions should be performed.

The analgesic drugs inhibited propulsion of endogenous fecal pellets in a dose-dependent manner, irrespective of the effect of the vehicles (but, in view of the "excitatory" effect of the vehicles used, inhibition of colonic propulsion seemed to be more robust for loperamide and WIN 55,212-2). We analyzed several functional parameters to characterize the effects of the analgesics. One of them, propulsion speed, was calculated both directly from the fluoroscopic recordings (PS) and indirectly from the STM (PS'). Changes associated with increasing drug doses run in parallel, suggesting that both methods are valid.

Inhibition of intestinal motor function is a well-known effect of opioids and cannabinoids that is observed in both humans and animals. ${ }^{3,5}$ High doses of morphine are not only associated with nausea and vomiting, but also with constipation, as its major side effect., ${ }^{2,4}$ Loperamide also slows down intestinal peristaltic activity, leading to increased transit time, ${ }^{48,49}$ and it is mainly used in the clinic to treat diarrhea, due to its constipating effects. ${ }^{50,51}$ It is interesting to note that at the oral therapeutic doses used in the clinic to treat diarrhea, glycoprotein $\mathrm{P}$ (located in the gut barrier and the blood brain barrier) avoids loperamide to access the central nervous system, but higher oral (or systemic) doses may saturate glycoprotein $\mathrm{P}$ and exert central effects, ${ }^{52}$ including analgesia (unpublished observations from our own laboratory). At lower doses, it has also been demonstrated that loperamide may act on peripheral opioid receptors to reduce nociception in rat muscle pain models. ${ }^{20}$ Constipation is also a well-known effect of cannabis and its derivatives that has been traditionally used in the treatment of different gastrointestinal disorders. ${ }^{53}$ Specifically, the natural agonist delta-9-tetrahydrocannabinol has been shown to slow down intestinal transit and colonic propulsion in rodents, ${ }^{54,55}$ and we have extensively described the inhibitory effects of WIN 55,212-2, the synthetic non-selective cannabinoid agonist used in this report, on gastrointestinal motility., ${ }^{5,10,11,56,57}$

The doses used in this work were similar to those previously used by us and other researchers. ${ }^{9,10,48,49,57}$ Morphine and loperamide were used at $0.1,1,5$, and $10 \mathrm{mg} / \mathrm{kg}$, but WIN 55,212-2 was not tried at $10 \mathrm{mg} / \mathrm{kg}$ due to solubility problems. However, results for WIN 55,212-2 were also dose-dependent and comparable to those obtained for the opioid drugs.

Although, in general, the higher the dose, the more intense inhibition of colonic motor function, it is noteworthy that in animals treated with the lowest dose of morphine (MOR 0.1), functional parameters did not fit with the general tendency and colonic motor function was stimulated (instead of being inhibited). Interestingly, it has been demonstrated that very low doses of morphine intravenously administered produces maximum stimulation of duodenal contractility in healthy volunteers. ${ }^{58}$ In fact, excitatory effects of drugs that normally exert inhibitory actions are frequently encountered. Thus, other inhibitors of the central nervous system used at low doses also stimulate (instead of inhibiting) brain functions (alcohol as an example ${ }^{59}$ ). The fact that loperamide, the other opioid agonist tested, did not exert this stimulatory effect at low doses suggests that it might be more related to a central action of the drug, since at low doses loperamide does not cross the blood-brain barrier and thus, it does not induce central effects. ${ }^{20,60}$ In the case of WIN, no consistent central (psychoactive) effects were detected at doses of $1 \mathrm{mg} / \mathrm{kg}$ in the cannabinoid tetrad, ${ }^{21,23,46,61}$ suggesting that this drug at low doses might also be devoid of excitatory effects, not only on colonic motor function but also in the central nervous system.

In conclusion, colonic propulsion of endogenous fecal pellets was studied using fluoroscopy and STM in conscious rats. This method was able to show the well-known dose-dependent effects of different analgesic drugs on colonic motor function. Future research will be devoted to evaluate the effects of other drug treatments and physiopathological situations on colon motor function without the interference of anesthetics.

\section{Supplementary Materials}

Note: To access the supplementary figures and videos mentioned in this article, visit the online version of Journal of Neurogas- 
troenterology and Motility at http://www.jnmjournal.org/, and at https://doi.org/10.5056/jnm18202.

Financial support: This work was supported by Ministerio de Ciencia e Innovación (SAF2009-12422-C02-01 and SAF201240075-C02-01) and Comunidad de Madrid (S-SAL/0261/2006 and S2010/BMD-2308).

\section{Conflicts of interest: None.}

Author contributions: Raquel Abalo designed the study. Susana Díaz-Ruano, Ana E López-Pérez, Rocío Girón, Irene PérezGarcía, and Raquel Abalo performed the experiments; Susana Díaz-Ruano analyzed the data; Susana Díaz-Ruano, Ana E LópezPérez, and Raquel Abalo wrote the manuscript; and María I Martín-Fontelles contributed financial support and essential intellectual input. All authors critically reviewed and approved the final version of the manuscript, and agree to be accountable for all aspects of the work.

\section{References}

1. Nelson D, Camilleri M. Opioid-induced constipation: advances and clinical guidance. Ther Adv Chronic Dis 2016;7:121-134.

2. Kalso E, Edwards JE, Moore RA, McQuay HJ. Opioids in chronic non-cancer pain: systematic review of efficacy and safety. Pain 2004;112:372-380.

3. Oosten AW, Oldenmenger WH, Manthijssen RH, van der Ritj CC. A systematic review of prospective studies reporting adverse events of commonly used opioids for cancer-related pain: a call for the use of standardized outcome measures. J Pain 2015;16:935-946.

4. Kumar L, Baker C, Emmanuel A. Opioid-induced constipation: physiopathology, clinical consequences, and management. Gastroenterol Res Pract 2014:141737.

5. Abalo R, Vera G, López-Pérez AE, Martínez-Villaluenga M, MartínFontelles MI. The gastrointestinal pharmacology of cannabinoids: focus on motility. Pharmacology 2012;90:1-10.

6. Lichtenberger LM, Bhattarai D, Phan TM, Dial EJ, Uray K. Suppression of contractile activity in the small intestine by indomethacin and omeprazole. Am J Physiol Gastrointest Liver Physiol 2015;308:G785G793.

7. Schereiber D, Klotz M, Laures K, Clasohm J, Bischof M, Schäfer KH. The mesenterially perfused rat small intestine: a versatile approach for pharmacological testings. Ann Anat 2014;196:158-166.

8. Torjman MC, Joseph JI, Munsick C, Morishita M, Grunwald Z. Effects of isofluorane on gastrointestinal motility after brief exposure in rats. Int J Pharm 2005;294:65-71.

9. Girón R, Pérez-García I, Abalo R. X-ray analysis of gastrointestinal motility in conscious mice. Effects of morphine and comparison with rats. Neurogastroenterol Motil 2016;28:74-84.

10. Abalo R, Cabezos PA, Vera G, Fernández-Pujol R, Martín MI. The cannabinoid antagonist SR144528 enhances the acute effect of WIN $55,212-2$ on gastrointestinal motility in the rat. Neurogastroenterol Motil 2010;22:694-e206.

11. Abalo R, Cabezos PA, López-Miranda V, et al. Selective lack of tolerance to delayed gastric emptying after daily administration of WIN 55,212-2 in the rat. Neurogastroenterol Motil 2009;21:1002-e80.

12. Costa M, Dodds KN, Wiklendt L, Spencer NJ, Brookes SJ, Dinning PG. Neurogenic and myogenic motor activity in the colon of the guinea pig, mouse, rabbit, and rat. Am J Physiol Gastrointest Liver Physiol 2013;305:G749-G759.

13. Costa M, Wiklendt L, Simpson P, Spencer NJ, Brookes SJ, Dinning PG. Neuromechanical factors involved in the formation and propulsion of fecal pellets in the guinea-pig colon. Neurogastroenterol Motil 2015;27:1466-1477.

14. Huizinga JD, Chen JH, Zhu YF, et al. The origin of segmentation motor activity in the intestine. Nat Commun 2014;5:3326.

15. Ramírez I, Pantrigo JJ, Montemayor AS, et al. Computer vision-based diameter maps to study fluoroscopic recordings of small intestinal motility from conscious experimental animals. Neurogastroenterol Motil Published Online First 16 Mar 2017. doi: 10.1111/nmo.13052.

16. López-Gómez L, Díaz-Ruano S, Girón R, et al. Preclinical evaluation of the effects on the gastrointestinal tract of the antineoplastic drug vincristine repeatedly administered to rats. Neurogastroenterol Motil 2018;30:e13399.

17. Trescot AM, Glaser SE, Hansen H, Benyamin R, Patel S, Manchikanti L. Effectiveness of opioids in the treatment of chronic non-cancer pain. Pain Physician 2008;11(2 suppl):S181-S200.

18. De Schepper HU, Cremonini F, Park MI, Camilleri M. Opioids and the gut: pharmacology and current clinical experience. Neurogastroenterol Motil 2004;16:383-394.

19. Baker DE. Loperamide: a pharmacological review. Rev Gastroenterol Disord 2007;7(suppl 3):S11-S18.

20. Sánchez EM, Bagües A, Martín MI. Contributions of peripheral and central opioid receptors to antinociception in rat muscle pain models. Pharmacol Biochem Behav 2010;96:488-495.

21. Vera G, Cabezos PA, Martín MI, Abalo R. Characterization of cannabinoid-induced relief of neuropathic pain in a rat model of cisplatin-induced neuropathy. Pharmacol Biochem Behav 2013;105:215-212.

22. Bridges D, Ahmad K, Rice AS. The synthetic cannabinoid WIN55, 212-2 attenuates hyperalgesia and allodynia in a rat model of neuropathic pain. Br J Pharmacol 2001;133:586-594.

23. Pascual D, Goicoechea C, Suardíaz M, Martín MI. A cannabinoid agonist, WIN 55,212-2, reduces neuropathic nociception induced by paclitaxel in rats. Pain 2005;118:23-34.

24. Khansari M, Sohrabi M, Zamani F. The useage of opioids and their adverse effects in gastrointestinal practice: a review. Middle East J Dig Dis 2013;5:5-16.

25. Furness JB. The enteric nervous system. Blackwell publishing. Melboume, Australia 2006. 
26. Wyse CA, McLellan J, Dickie AM, Sutton DG, Preston T, Yam PS. A review of methods for assessment of the rate of gastric emptying in the dog and the cat: 1898-2002. J Vet Intern Med 2003;17:609-621.

27. Camilleri M, Linden DR. Measurement of gastrointestinal and colonic motor functions in humans and animals. Cell Mol Gastroenterol Hepatol 2016;2:412-428.

28. Cannon WB. Movements of the stomach, studied by means of the Röntgen rays. J Boston Soc Med Sci 1898;2:59.

29. Marciani L. Assessment of gastrointestinal motor functions by MRI: a comprehensive review. Neurogastroenterol Motil 2011;23:399-407.

30. Hertz AF, Morton CJ, Cook F. The passage of food along the human alimentary canal. Guy's Hosp Rep 1907;61:389-427.

31. Ritchie JA, Truelove SC, Ardan GM, Tuckey MS. Propulsion and retropulsion of normal colonic contents. Am J Dig Dis 1971;16:697-704.

32. Ehrlein HJ, Reich H, Schwinger M. Colonic motility and transit of digesta during hard and soft faeces formation in rabbits. J Physiol 1983;338:75-86

33. Bedrich M, Ehrlein H. Motor function of the large intestine and flow of digesta in sheep. Small Rumiant Research 2001;42:141-154.

34. Hipper K, Ehrlein HJ. Motility of the large intestine and flow of digesta pigs. Rev Vet Sci 2001;71:93-100.

35. Myagmarjalbuu B, Moon MJ, Heo SH, et al. Establishment of protocol for determining gastrointestinal transit time in mice using barium and radiopaque markers. Korean J Radiol 2013;14:45-50.

36. Bouchoucha M, Benard T, Dupres M. Temporal and spatial rhythmicity of jejunal wall motion in rats. Neurogastroenterol Motil 1999;11:339346.

37. Henning GW, Costa M, Chen BN, Brookes SJ. Quantitative analysis of peristalsis in the guinea-pig small intestine using spatio-temporal maps. J Physiyol 1999;517(Pt 2):575-590.

38. Bercík P, Bouley L, Dutoit P, Blum AL, Kucera P. Quantitative analysis of intestinal motor patterns: spatiotemporal organization of nonneural pacemarker sites in the rat ileum. Gastroenterology 2000;119:386-394.

39. Keding DM, Hurst NR, Grider JR. Spatiotemporal mapping of motility in ex vivo preparations of the intestines. J Vis Exp 2016:e53263.

40. Barnes KJ, Beckett EA, Brookes SJ, Sia TC, Spencer NJ. Control of intrinsic pacemaker frequency and velocity of colonic migrating motor complexes in mouse. Front Neurosci 2014;8:96.

41. Arkwright JW, Underhill ID, Dodds KN, et al. A composite fibre optic catheter for monitoring peristaltic transit of an intra-luminal bead. J Biophotonics 2016;9:305-310.

42. Ailiani AC, Neuberger T, Brasseur JG, et al. Qunatifying the effects of inaction vs isoflurane anesthesia on gastrointestinal motility in rats using dynamic magnetic resonance imaging and spatio-temporal maps. Neurogastroenterol Motil 2014;26:1477-1486.

43. Der T, Bercik P, Donnelly G, et al. Interstitial cells of Cajal and inflammation-induced motor dysfunction in the mouse small intestine. Gastroenterology 2000;119:1590-1599.

44. Wuang XY, Lammers WJ, Bercik P, Huizinga JD. Lack of pyloric interstitial cells of Cajal explains distinct peristaltic motor patterns in stomach and small intestine. Am J Physiol Gastrointest Liver Physiol 2005;289:G539-G549.
45. McQuade RM, Stojanovska V, Donald EL, et al. Irinotecan-induced gastrointestinal dysfunction is associated with enteric neuropathy, but increased numbers of cholinergic myenteric neurons. Front Physiol 2017;8:391.

46. Vera G, Chiarlone A, Cabezos PA, Pascual D, Martín MI, Abalo R. WIN 55,212-2 prevents mechanical allodynia but not alterations in feeding behavior induced by chronic cisplatin in the rat. Life Sci 2007;81:468479 .

47. Pestel S, Martin HJ, Maier GM, Guth B. Effect of commonly used vehicles on gastrointestinal, renal, and liver function in rats. J Pharmacol Toxicol Methods 2006;54:200-214.

48. Tan-No K, Niijima F, Nakagawasai O, Sato T, Satoh S, Tadano T. Development of tolerance to the inhibitory effect of loperamide on gastrointestinal transit in mice. Eur J Pharm Sci 2003;20:357-363.

49. Padmanabhan P, Grosse J, Asad AB, Radda GK, Golay X. Gastrointestinal transit measurements in mice with ${ }^{99 \mathrm{~m}} \mathrm{Tc}$-DTPA-labeled activated charcoal using NanoSPECT-CT. EJNMMI Res 2013;3:60.

50. Ericsson CD, Johnson PC. Safety and efficacy of loperamide. Am J Med 1990;88(6A):10S-14S.

51. Wu PE, Juurlink DN. Clinical review: loperamide toxicity. Ann Emerg Med 2017;70:245-252.

52. Schinkel AH, Wagenaar E, Mol CA, van Deemter L. P-glycoprotein in the blood-brain barrier of mice influences the brain penetration and pharmacological activity of many drugs. J Clin Invest 1996;97:2517-2524.

53. Izzo AA, Coutts AA. Cannabinoid and the digestive tract. Handb Exp Pharmacol 2005:573-598.

54. Chester GB, Dahl CJ, Everingham M, Jackson DM, MarchantWilliams H, Starmer GA. The effect of cannabinoids on intestinal motility and their antinociceptive effect in mice. Br J Pharmacol 1973;49:588594.

55. Aviello G, Romano B, Izzo AA. Cannabinoids and gastrointestinal motility: animal and human studies. Eur Rev Med Pharmacol Sci 2008;12(suppl 1):81-93.

56. Abalo R, Cabezos PA, Vera G, López- Miranda V, Herradón E, MatínFontelles MI. Cannabinoid-induced delayed gastric emptying is selectively increased upon intermittent administration in the rat: role of $\mathrm{CB} 1$ receptors. Neurogastroenterol Motil 2011;23:457-467, e177.

57. Abalo R, Cabezos PA, Vera G, López-Pérez AE, Martín MI. Cannabinoids may worsen gastric dysmotility induced by chronic cisplatin in the rat. Neurogastroenterol Motil 2013;25:373-382, e292.

58. Lewis TD. Morphine and gastroduodenal motility. Dig Dis Sci 1999;44:2178-2186

59. Arizzi-LaFrance MN, Correa M, Aragon CM, Salamone JD. Motor stimulant effects of ethanol injected into the substantia nigra pars reticulata: importance of catalase-mediated metabolism and the role of acetaldehyde. Neuropsychopharmacology 2006;31:997-1008.

60. Eyal S, Hsiao, Unadkat JD. Drug interactions at the blood-brain barrier: fact or fantasy? Pharmacol Ther 2009;123:80-104.

61. Vera G, López-Miranda V, Herradón E, Martín MI, Abalo R. Characterization of cannabinoid-induced relief of neuropathic pain in rat models of type 1 and type 2 diabetes. Pharmacol Biochem Behav 2012;102:335343. 